\title{
Anabases
}

ANABASES Traditions et réceptions de l'Antiquité

32 | 2020

Varia

\section{Mark A. Ralkowski, Plato's Trial of Athens}

Jean Zaganiaris

\section{OpenEdition}

Journals

Édition électronique

URL : https://journals.openedition.org/anabases/11547

DOI : 10.4000/anabases. 11547

ISSN : 2256-9421

\section{Éditeur}

E.R.A.S.M.E.

\section{Édition imprimée}

Date de publication : 20 octobre 2020

Pagination : 278-280

ISSN : 1774-4296

\section{Référence électronique}

Jean Zaganiaris, " Mark A. Ralkowski, Plato's Trial of Athens », Anabases [En ligne], 32 | 2020, mis en ligne le 20 octobre 2020, consulté le 15 novembre 2022. URL : http://journals.openedition.org/ anabases/11547 ; DOI : https://doi.org/10.4000/anabases.11547 

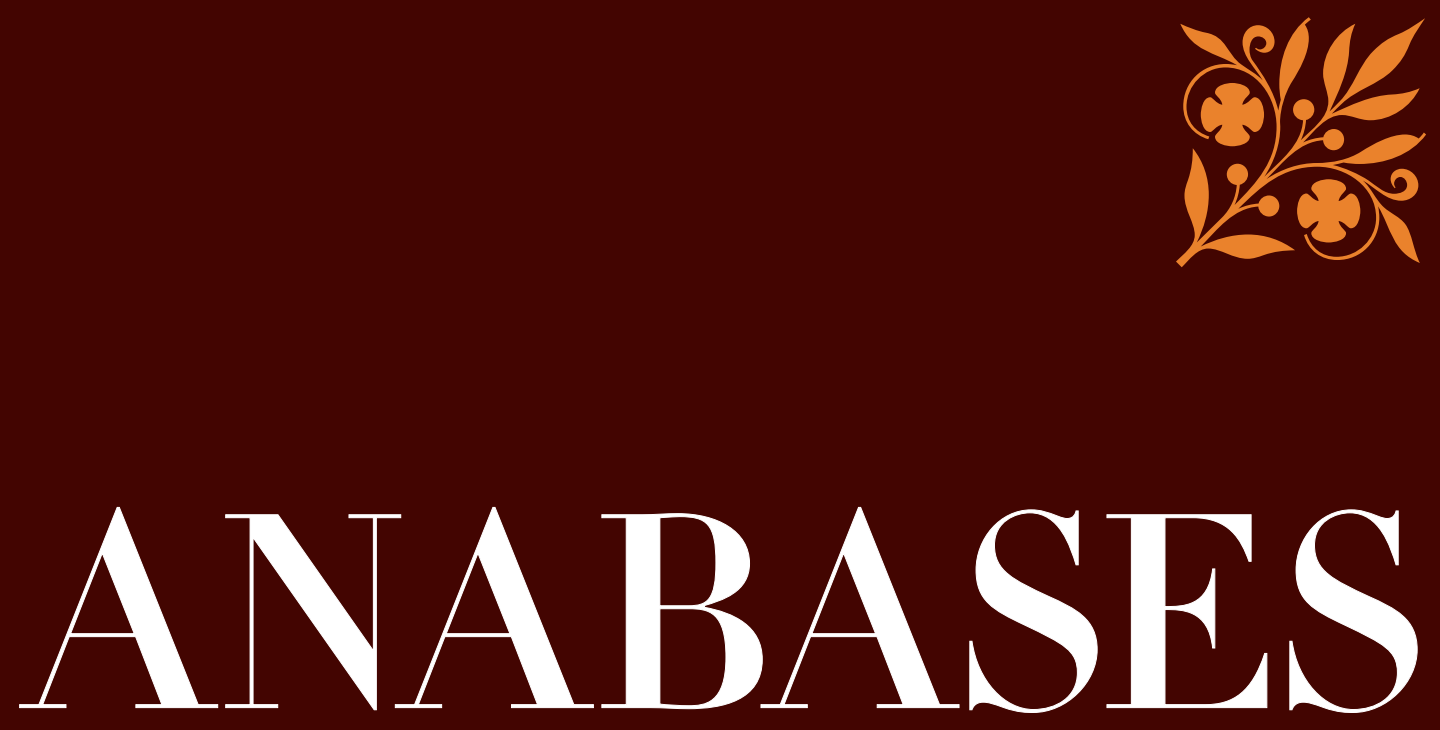

Traditions et Réceptions de l'Antiquité

\section{No32 \\ 2020}

Antiquaires ef archéologues d'antan L'histoire romaine et le politique Relire Gsell L'Invention du Théâtre Antique dans les paratextes savants El imaginario prehistórico y la Antigüedad en el cine 
ANABASES

Traditions et Réceptions de l'Antiquité

Revue de l'équipe de recherche E.R.A.S.M.E.

Université Toulouse-Jean Jaurès (UT2J)

Anabases dispose d'un Comité de lecture international. Chaque article envoyé à la rédaction est soumis, une fois anonymisé, à l'expertise de deux spécialistes qui rendent un rapport écrit. Les deux rapports anonymisés sont transmis à l'auteur qui tient compte des observations en vue de la publication.

\section{Comité SCIENTIFique}

Germaine Aujac (université Toulouse-Jean Jaurès : histoire de la géographie et des sciences antiques)

Florence Bouchet (université Toulouse-Jean Jaurès : littérature médiévale)

Hinnerk Brunns (CNRS : histoire économique et sociale ancienne et contemporaine)

Paulo Butti de Lima (université de Bari : historiographie et réception de l'Antiquité)

Luciano CANFora (université de Bari : littérature et histoire anciennes, historiographie)

Giovanna Ceserani (Stanford University : histoire intellectuelle et historiographie de la tradition classique)

Temístocles Cezar (université de Porto Alegre : historiographie moderne)

Serafina Сuомо (University of London, Birkbeck College : histoire des mathématiques et des sciences)

Paul Demont (université de Paris Sorbonne : philologie grecque et héritage classique)

Marie-Laurence Desclos (université de Grenoble II : philosophie de l'Antiquité)

Olivier Devillers (université de Bordeaux 3 - Michel-de-Montaigne : littérature et historiographie latines)

Andrea Giardina (Istituto italiano di scienze umane : histoire du monde romain et de ses réceptions)

Ève Gran-Aymerich (AIBL : histoire de l'archéologie et des transferts culturels)

François Hartog (EHEss : historiographie ancienne et moderne)

Geneviève Hoffmann (université de Picardie : histoire des mondes grecs)

Christian JACOB (CNRS/EHESS : histoire comparée et épistémologie des savoirs)

Suzanne Marchand (Louisiana State University : histoire du classicisme et de l'orientalisme)

Wilfried NIPPEL (Humboldt Universität Berlin : histoire et historiographie de l'Antiquité)

Sylvie Pitria (université de Paris I-Panthéon Sorbonne : histoire et historiographie du monde romain)

Stéphane Ratтi (université de Franche-Comté - Besançon : philologie et héritage latin)

Comité de RÉdACtion

Clément Bertau-Courbières, Corinne Bonnet, Laurent bricault, Clément Bur, Adeline Grand-Clément, Anne-Hélène Klinger-Dollé, Véronique Krings, Thibaud Lanfranchi, Pascal Payen, Grégory Reimond, Sarah Rey, Catherine Valenti, Noémie Villacèoue

Éditeur RESPONSABLE

Clément Bur

Éditrice ADJOINTE

Catherine VALENTI

Sites WEB

http://plh.univ-tlse2.fr

Revues.org : http://anabases.revues.org

Aвonnement et vente aU numéro

Éditions De Boccard - 4, rue de Lanneau - 75005 Paris

info@deboccard.com - www.deboccard.com

Tél. : 0033/(0)143260037 - Fax : 0033/(0)143548583 


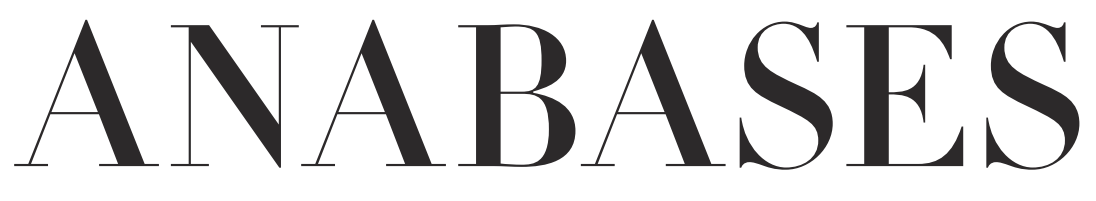

Traditions et Réceptions de l'Antiquité

$$
\begin{aligned}
& N \circ 32 \\
& 2020
\end{aligned}
$$

\section{E.R.A.S.M.E.}

Université Toulouse - Jean Jaurès 



\section{Sommaire}

$\mathrm{N}^{\circ} 32$ - 2020

\section{Historiographie et identités culturelles}

\section{Pascal Montlahuc}

L'histoire romaine et le politique : complément d'enquête . . . . . . . . II

\section{Traditions du patrimoine antique}

\section{Alberto Lombo Montañés}

Apuntes para el estudio del imaginario prehistórico y sus relaciones con la Antigüedad clásica en los personajes del cine moderno . . . . . . . 33

Jorge Elices Ocón

Antiquity depicted as part of a discourse:

from the first European travellers to the DAESH propaganda . . . . . . . . 49

\section{Archéologie des savoirs}

Dossier Antiquaires \& archéologues d'antan

(dossier dirigé par Vivien Barrière)

Vivien Barrière \& Véronique Krings

Introduction . . . . . . . . . . . . . . . . $7^{3}$

Alessia Zambon

Acquisition et transmission du savoir antiquaire à Athènes

à la fin du XVIII ${ }^{\mathrm{e}}$ et au début du XIX ${ }^{\mathrm{e}}$ siècle :

Fauvel et son réseau de sociabilité . . . . . . . . . . . . . . 79 
Christian MazeT

Les antiques de Gustave-Adolphe Beugnot (I799-186I).

Histoire et fortune d'une collection oubliée . . . . . . . . . . . . . . IO7

Vivien BARRIÈRE

Les Antiques d'Autun à la Renaissance : poids des travaux historiques et émergence d'un discours antiquaire $(\mathrm{r} 529-\mathrm{I} 620) \quad \ldots \ldots$. . . . . . . . I33

Marie-Laurence HAACK

Les Étrusques et la question raciale dans l'Italie fasciste . . . . . . . . I5 I

Anne-Julie Eтter

Temples et pyramides : la place de l'Égypte dans l'étude

des antiquités de l'Inde $\left(\mathrm{xVIII}^{\mathrm{e}}-\mathrm{xIXX}^{\mathrm{e}}\right.$ siècle $) \ldots \ldots \ldots \ldots \ldots$. . . . . . . . . .

\section{Actualités et débats}

Malika Bastin-Hammou et Pascale Paré

- ITHAC - L'Invention du Théâtre Antique dans le Corpus des paratextes savants du xvi ${ }^{\mathrm{e}}$ siècle. Analyse, traduction, exploration numérique . . . . . i 85

\section{Lire, relire la bibliothèque des sciences de l'Antiquité}

\section{Philippe Leveau}

Lectures coloniales et post-coloniales de l'histoire de l'Afrique romaine.

Histoire et constructions mémorielles. À propos de Stéphane Gsell,

" La Tripolitaine et le Sahara au II $^{\mathrm{e}}$ siècle de notre ère ",

Mémoires de l'Académie des Inscriptions et Belles-Lettres,

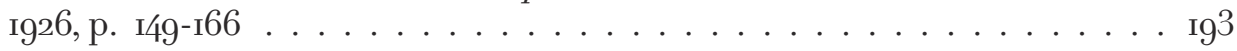

Stéphane Gsell

" La Tripolitaine et le Sahara au III siècle de notre ère ",

Mémoires de l'Académie des Inscriptions et Belles-Lettres, Ig26, p. I49-I66 . . 2II

\section{Ateliers}

Antiquités numériques (coordonné par E. Guillon) ( $\left.{ }^{\circ} 3\right)$

Jean-Louis Ferrary et Philippe Moreau

La base de données $L E P O R$. . . . . . . . . . . . . . . . . . . 23I

Actualités du théâtre $\left(n^{\circ} 6\right)$

Catherine Ailloud-Nicolas

Se méfier des classiques ? À propos d'Oreste à Mossoul de Milo Rau . . . . 2377 
Archives de savant $\left(n^{\circ} 15\right)$

Massimo Cultraro

Heinrich Schliemann e l'Italia: storie di archivi, narrazioni di uomini . . . 240

\section{Comptes rendus}

Antony Augoustakis et Stacie Raucci (éds.)

Epic Heroes on Screen (Mathieu Scapin) . . . . . . . . . . . . . . . . . . 253

Patrick Baker, Johannes Helmrath et Craig Kallendorf (éds.)

Beyond Reception: Renaissance Humanism

and the Transformation of Classical Antiquity (Alexia Dedieu) . . . . . . . . 255

Dominique Millet-GÉrard

Édition de Jacob Balde,

Jephtias Tragoedia / La Fille de Jephté, tragédie (Simone de Reyff) . . . . . 257

Joshua Billings, Felix Budelmann et Fiona Macintosh (éds.)

Choruses, Ancient and Modern (Malika Bastin-Hammou) . . . . . . . . . . . 259

Tiphaine Annabelle Besnard et Mathieu Scapin (dir.)

Age of Classics! L'Antiquité dans la culture pop.

Catalogue de l'exposition présentée au Musée Saint-Raymond,

Musée d'archéologie de Toulouse, du 22 février au 22 septembre 2019

(Élodie Guillon) . . . . . . . . . . . . . . . . . . . . 260

Fiona Cox

Ovid's Presence in Contemporary Women's Writing.

Strange Monsters (Séverine Tarantino) . . . . . . . . . . . . . . . . . . 262

Annick Fenet, Michela Passini et Sara Nardi-Combescure (dir.)

Hommes et patrimoines en guerre. L'heure du choix (IgI4-IgI8)

(Natacha Lubtchansky) . . . . . . . . . . . . . . . . . . . . . . . 264

Kristopher F. B. Fletcher et Osman Umurhan (éd.)

Classical Antiquity in Heavy Metal Music

(Francesco Xella et Corinne Bonnet) . . . . . . . . . . . . . . . . . . . 266

Shawn W. FLYNN (éd.)

Children in the Bible and the Ancient World.

Comparative and Historical Methods in Reading Ancient Children

(Sophie Laribi Glaudel) . . . . . . . . . . . . . . . . . . . . . . . . 267

Erminio Fonzo

Il mondo antico negli scritti di Antonio Gramsci (Andrea Avalli) . . . . . . 269 
Patrick Gray

Shakespeare and the Fall of the Roman Republic. Selfhood,

Stoicism and Civil War (Cyrielle Landrea) . . . . . . . . . . . . . . . . 270

Mélanie Lozat et Sara Petrella (dir.)

La Plume et le calumet. Joseph-François Lafitau

et les « sauvages ameriquains» (Clément Bur) . . . . . . . . . . . . . . 27I

Bartolo Natoli et Steven Hunt (éds.)

Teaching Classics with Technology (Léa Marques) . . . . . . . . . . . . . . . 274

S. Douglas Olson (éd.)

Ancient Comedy and Reception.

Essays in Honor of Jeffrey Henderson (Malika Bastin-Hammou) . . . . . . . 276

Mark A. Ralkowski

Plato's Trial of Athens (Jean Zaganiaris) . . . . . . . . . . . . . . . . . 278

Brett M. Rogers et Benjamin Eldon Stevens (ed.)

Once and Future Antiquities

in Science Fiction and Fantasy (Clément Bur) . . . . . . . . . . . . . . . . 280

Stine Schierup (éd.)

Documenting Ancient Rhodes: Archaeological Expeditions

and Rhodian Antiquities (Filippo Carlà-Uhink) . . . . . . . . . . . . . . . . 282

Vasiliki Zachari, Élise Lehoux et Noémie Hosoi (dir.)

La cité des regards. Autour de François Lissarrague

(Clarisse Evrard) . . . . . . . . . . . . . . . . . . . . . 284

Sandra ZANELLA

La caccia fu buona : pour une histoire des fouilles à Pompéi

de Titus à l'Europe (Vivien Barrière) . . . . . . . . . . . . . . . . . . . . 286

Donna Zuckerberg

Not All Dead White Men. Classics and Misogyny in the Digital Age

(Fabien Bièvre-Perrin) . . . . . . . . . . . . . . . . . . . . 287

Résumés . . . . . . . . . . . . . . . . . . 29I

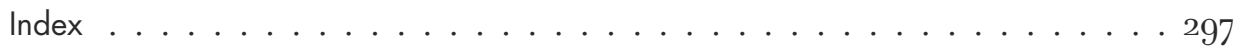




\section{Comptes rendus de lecture}



Antony Augoustakis et Stacie Raucci (éds.), Epic Heroes on Screen, Edinburgh, Edinburgh University Press, 20I8, 288 p., £8o / ISBN 978I47444245i6 (hardback), 20I9, £24,99 / ISBN 978I474454636 (paperback).

L'Antiquité au cinéma et à la télévision n'a jamais autant été étudiée que ces vingt dernières années. La raison principale est, bien entendu, le nombre de productions audiovisuelles en constante augmentation jusqu'en 20I4. Dans ce contexte, avouons que les chercheurs les plus prolifiques restent les Britaniques et les Nord-Américains.

Une nouvelle série d'ouvrages consacrée conjointement aux films et séries fait son apparition, Screening Antiquity, pour laquelle les éditeurs du premier volume, A. Augoustakis et S. Raucci, ont l'ambition de proposer des ouvrages à la pointe de la recherche académique (cutting-edge) sur la réception de l'Antiquité, à la fois sur le petit et le grand écran. Epic Heroes on Screen constitue un recueil d'actes d'une journée de conférences internationales, New Heroes on Screen, tenue à Delphes en 20I5. L'ensemble des participants et des auteurs des textes sont des chercheurs plus ou moins rompus à ce travail de recherche comme J.Solomon ou L. LlewellynJones par exemple. Le présent volume est séquencé en trois parties thématiques: Hercule d'abord, les héros épiques ensuite, les antihéros pour finir.

À la date à laquelle se tient le colloque à Delphes, deux films et un direct-to-video sur le héros Hercule ont été distribués l'année précédente, ce qui suffit à justifier qu'une partie des propositions soient axées sur le demi-dieu gréco-romain. L'article de J. Solomon revient nécessairement sur la filmographie d'Hercule en omettant, on pourra lui pardonner, les productions européennes d'avant l'ère Steve Reeves. Les trois auteurs suivants, A. Blanshard, E. Stafford et A. Chiu, s'intéressent aux productions de 2014: Hercules de Brett Ratner, The Legend of Hercules de Renny Harlin et le téléfilm de série Z Hercules Reborn de Nick Lyon. Globalement, les analyses s'orientent sur le traitement très “moderne» du personnage qui l'éloigne du demi-dieu mis en scène entre ig58 et les années I980. On regrettera les redites inhérentes d'un article à l'autre et le manque de réflexion sur l'importance de ces créations inter-médiatiques (du comics de Steve Moore au film de Ratner par exemple) ou les éléments qui nous semblent prépondérants sur ce personnage: dans les années ig6o, Hercule est présenté comme un demi-dieu, en 20I4, c'est avant tout un homme construit sur le modèle d'un avatar de jeu vidéo qui apprend avec l'expérience ou avec l'aide de personnages annexes parfois aussi importants que lui. Ce dernier argument est d'ailleurs soulevé par A. Blanshard mais de manière trop rapide, alors qu'il démontre très bien qu'à l'écran, ce n'est pas Hercule que l'on remarque d'abord mais l'acteur que la plastique rapproche justement de Steve Reeves: Dwayne “ The Rock » Johnson. E. Stafford, quant à elle, expose les œuvres d'un artiste pro-Poutine, qui représente le président russe en Hercule accomplissant ses Douze 
Travaux. Ainsi, elle introduit la notion de reprise politique déjà préexistante dans l'Antiquité et de manière continue tout au long de l'Histoire: l'Empereur Commode, le Tétrarque Maximien ou encore Henri IV. A. Chui s'intéresse à l'importance des compagnons dans le récit du film de Ratner: elle montre, mais de manière trop timide, combien le Hercule actuel au cinéma est plus un homme qu'un demidieu. Cette première partie se conclut sur une ouverture de M. E. Safran sur la thématique Hercule, à partir de la série américaine Supernatural, dans laquelle l'auteure montre que la mythologie d'Hercule est sous-jacente à celle du héros Dean Winchester, moins Jésus qu'Héraklès alors que les antagonistes sont tour à tour des anches déchus et des monstres proches du bestiaire monothéiste. Si, individuellement, chaque article apporte un éclairage intéressant sur la figure d'Hercule, on regrettera néanmoins la fragmentation des informations pourtant essentielles sur la façon dont le storytelling des années 2010 réinterprète Hercule au cinéma notamment.

La deuxième partie s'intéresse à la figure des héros épiques et les modèles à partir desquels ils sont construits puis montrés à l'écran. Le premier article, celui de M. S. Cyrino, est très intéressant. Il expose des théories nouvelles en matière d'étude de personnages antiques au cinéma mais nous a paru bien trop court. Elle expose notamment le fait que Russell Crowe aurait joué intentionnellement le rôle de Noé dans le film éponyme d'Aronofsky de la même façon que Maximus dans Gladiator, afin que le public puisse s'intégrer facilement dans le récit (cela pourrait-il s'apparenter au “Syndrome Jack Sparrow » qui tourmente Johnny Deep dans ses films post-Pirates des Caraïbes ?). La résultante de ce phénomène permettrait donc aux spectateurs de voir en Noé un héros sans faille et aimant, alors que le personnage est plus sombre. Trois autres articles présentent la façon dont sont construits les héros Thésée (M. M. Toscano), Xena (A. K. Strong) et les personnages principaux de la série Atlantis (A. Potter). Les auteurs observent les renversements de perspectives dans lesquelles les protagonistes principaux n'agissent pas forcément de la façon dont on l'attendrait dans les cycles épiques mais plutôt selon des modèles contemporains aux spectateurs : ces films évacuent d'une certaine façon la quête du divin pour faire des héros des êtres humains déconnectés des enjeux supérieurs menés par les dieux et déesses antiques.

Notons que l'article d'A. K. Strong aurait mérité un développement supplémentaire pour présenter le personnage de Xena, que l'auteure définit comme "polyamoureux » mais qui, selon nous, s'explique davantage comme le choix assumé des créateurs de la série de mettre en avant la relation homosexuelle entre Xena et Gabrielle. La construction des relations entre Xena et ses différents partenaires servirait plutôt à exacerber, dans l'esprit du spectateur des années I990, les liens entre les deux personnages principaux féminins. Une vision plutôt audacieuse et assumée pour une production grand public de cette époque qui est, à mon sens, bien plus qu'un sous-texte queer défendu par A. Strong...

Enfin, l'article d'H. Gardner revient sur la construction du héros dans le film Centurion (20Io). Il est judicieusement placé en fin de chapitre, comme pour introduire la figure de l'antihéros qui n'agit pas comme on l'attendrait d'un récit épique : absence de sacrifice héroïque, idéologisation des thèmes, fuite en avant, absence de restauration d'un ordre moral...

La dernière partie est composée de quatre articles tout aussi divers mais présentant, dans leur ensemble, les mêmes productions cinématographiques: 300 de Snyder, Alexandre de Stone et Clash of The Titans de Letterier (remakes plus ou moins assumés de péplum des années ig5o-ig6o). D. Curley, dans le premier article, théorise 
les rôles joués par les antihéros dans le récit et emploie sa démarche pour films cités précédemment, ainsi que pour celui d'Hercules de Brett Ratner. Les exemples choisis correspondent à sa théorisation puisqu'elle s'applique à des histoires concentrées autour d'une figure majeure construite sur le modèle du « monomythe ». Il montre bien comment le film Alexandre d'Oliver Stone retourne complètement l'image de celui du film de Robert Rossen de ig56, tout comme le Clash of The Titans de 20 oro s'émancipe de celui de ig8I.

L. Llewellyn-Jones revient sur le cas $30 o$, et le destin qu'a connu ce film au Moyen-Orient, notamment en Iran. On regrettera cependant, comme à l'accoutumée, lorsqu'il est question d'une adaptation d'un comics, que l'analyse soit un peu trop décontextualisée de sa source dessinée et son auteur (dans la mesure où le film respecte plutôt graphiquement le comics): oui, le discours présenté dans 300 est problématique et dangereux sur tous les plans, mais l'analyse du film ne doit pas être dissociée de celle de l'œuvre de Miller, ouvertement néoconservateur et dont l'œuvre fait montre de son adhésion au lobby des armes et à la guerre en Irak et Afghanistan. 3oo s'inscrit dans un mouvement de création complètement bouleversé par les évènements du II septembre 200I comme le montre très bien l'article de Vincent Tomasso. En effet, les productions cinématographiques sont irrémédiablement transformées et montrent une vision bien différente de la société américaine et du monde. Dans le cadre du néo-péplum, ce changement s'opère avec Troie de Petersen (2004), qui amorce une réflexion sur la représentation du divin et des dieux au cinéma : c'est la fin de la pensée mythique, de l'hégémonie des êtres puissants non-humains. Pour l'auteur, le traumatisme mondial du ir septembre a obligé les Nord-Américains à se reposer la question de leur identité multiple. Le dernier article, celui d'A. McAuley, présente une thématique assez intéressante sur les rapports possibles à faire entre les biopics de personnages contemporains au cinéma et le mécanisme de la Laudatio funebris, en prenant notamment pour exemple celui de Steve Jobs.

L'ouvrage est complété d'une bibliographie bien fournie mais anglophone (mis à part deux travaux de Claude Aziza et un de Michel Éloy). Elle est le malheureux reflet des deux traditions de réception : l'anglosaxonne, déjà en avance sur la question de l'Antiquité au cinéma, très souvent publiée, prolifique (on ne compte plus le nombre de thèses soutenues sur la question), quitte à tourner en rond; la francophone, bouillonnante, en pleine expansion mais peu accessible du fait des publications trop rares. Cet Epic Heroes on Screen marque cette dichotomie: intéressant pour les non-spécialistes, parfois novateur sur certaines questions théoriques mais avec ce sentiment que les thèmes ont du mal à être renouvelés. Une lecture à conseiller malgré tout.

$$
\begin{array}{r}
\text { Mathieu ScapiN } \\
\text { Musée Saint-Raymond, Musée } \\
\text { d'archéologie de Toulouse } \\
\text { mathieu.scapin@gmail.com }
\end{array}
$$

Patrick Baker, Johannes Helmrath et Craig Kallendorf (éds.), Beyond Reception: Renaissance Humanism and the Transformation of Classical Antiquity, Berlin et Boston, De Gruyter, 2019, 208 p., 79,95 € / ISBN 9783iıo635775.

Entre 2005 et 20I7, le Centre de Recherches collaboratif “ Transformation de l'Antiquité » (le Sonderforschungsbereich 644), piloté par l'Université Humboldt de Berlin, a hébergé vingt-sept différents projets dont la visée était d'élaborer une nouvelle méthodologie pour d'aborder l'étude de la réception de l'Antiquité. 
Dans ce cadre s'est tenue en 2015 à Berlin une conférence dont est issu cet ouvrage. La nouvelle approche proposée vise à envisager la réception de l'Antiquité comme une "transformation". Alors que la réception met davantage l'accent sur la culture qui reçoit et interprète l'Antiquité, le concept de “transformation» insiste sur l'influence réciproque que peuvent avoir l'une sur l'autre l'Antiquité (sphère de référence) et la sphère de réception (l'époque postérieure qui procède à sa propre lecture de l'Antiquité).

La notion d'allélopoièse (“création réciproque»; p. 4), qui insiste sur la nécessité de se défaire d'une idée “unidirectionnelle » des dynamiques entre deux époques, est ainsi proposée pour étudier la “construction réciproque " à l'œuvre dans la transformation de l'Antiquité. Si cette création réciproque d'une époque au prisme de l'autre n'était jusqu'à aujourd'hui pas passée inaperçue dans les études de réception, le mérite revient à cet ouvrage d'en proposer une théorie qui permette de l'aborder de façon plus systématique. L'introduction se clôt sur le postulat que le développement de cette nouvelle méthodologie permettra l'éclosion d'une " nouvelle ère des études sur l'humanisme ", avançant ainsi l'hypothèse suivante: by focusing on transformation, we can update the foundational metaphor of the Renaissance and launch the study of humanism into a new age, in which active change replaces passive reception as the key to cultural understanding (p.3). Chaque chapitre explore donc les enjeux de cette allélopoièse inhérente aux travaux humanistes en se fondant sur une typologie présentée au chapitre 2, qui propose quatorze types de transformations et illustre chacune de ces définitions théoriques au moyen d'exemples.

Les trois premiers chapitres étudient des phénomènes de transformations qui impliquent des innovations en termes de pédagogie. Giancarlo Abbamonte (chap. 3) montre que Lorenzo Valla transforme l'en- seignement du latin en imposant le recours aux textes classiques dans leur ensemble, et répond ainsi à une précédente transformation de la langue par les scholastiques. S'il est bien connu que l'humanisme est caractérisé par une circulation des textes fondateurs de la littérature classique d'Est en Ouest, Federica Ciccolella (chap.4) s'attache à montrer que la culture humaniste a également circulé d'Ouest en Est. Ainsi, durant la Renaissance, la Crète est durablement marquée par l'influence de l'Ouest. Enfin, Peter Mack (chap. 5) étudie l'influence de Rudolph Agricola et Erasme sur la rhétorique, enseignée en plus étroite connexion avec les textes de littérature classiques. Ce renouveau est indissociable d'un renouvellement des méthodes d'enseignement du latin.

Lestrois chapitres suivants se concentrent sur des enjeux politiques et historiques. Johannes Helmrath (chap. 6), prenant pour exemple les discours d'Aeneas Piccolomini devant les Diètes, poursuit l'étude des transformations de la rhétorique antique opérées par les humanistes et les enjeux de cette transformation dans un cadre cette fois-ci politique. James Hankins (chap. 7) défend l'idée que la transformation des textes de philosophie ancienne contribue, dans la pensée humaniste, à la construction d'une politique de la vertu (virtue politics) présentée comme étant à l'origine des institutions politiques modernes (p. III). Roland Béhar (chap. 8) s'attache ensuite à démontrer que, dans le De viris illustribus et les Triumphi, Pétrarque transforme le rituel des triomphes. Ces transformations, à l'origine du développement de l'histoire en tant que discipline humaniste, ont également concouru au développement des pratiques archéologiques et épigraphiques à la fin du Quattrocento.

Les trois derniers chapitres s'approprient plus en détail le caractère allélopoiétique de la transformation et s'attachent à dépeindre comment les pratiques des savants humanistes ont contribué à 
inventer l'Antiquité. Craig Kallendorf (chap. 9) montre que l'œuvre de Virgile est à la Renaissance le lieu où s'élabore une vision idéalisée de l'Antiquité. Il illustre les multiples transformations que connaissent les œuvres du poète à la Renaissance et peut ainsi conclure : Virgil's humanist readers constructed the understanding of the classical past that they thought they were simplyrecovering (p. I47). Jill Kraye (chap. Io) dresse un panorama des différentes transformations, intentionnelles ou non, opérées par les humanistes sur les philosophies antiques. Elle illustre ainsi la diversité des orientations et évolutions qu'ont connues l'aristotélisme, le platonisme, le stoïcisme, l'épicurisme et le scepticisme. Enfin, s'opposant à l'idée qui voudrait que les humanistes ne soient pas des philosophes, Ada Palmer (chap. II) prouve que les types de transformations des auteurs classiques mis en œuvre dans les textes ont évolué entre la période humaniste et le xvII ${ }^{\mathrm{e}}$ siècle. Car les humanistes, qui dissimulaient leurs innovations sous des " masques classiques » (classicalmasks, p. I77), n'en revendiquaient pas l'originalité. Elle démontre ainsi que ces divergences ont orienté les perceptions que l'on a pu avoir de ces époques et de leurs contributions à l'histoire de la littérature et de la philosophie jusqu'à aujourd'hui, au profit des philosophies plus récentes. Ada Palmer conclut sur une défense de la pertinence du concept de transformation: Transformation theory can help us differentiate the various strategies for transforming classical material that have had in turn such a profound impact on the way earlier Renaissance and seventeenthcentury thinkers are perceived today (p. I78).

La variété des analyses proposées par cet ouvrage répond de façon convaincante au but que s'était fixé l'introduction et illustre l'intérêt que peut revêtir cette notion de transformation. La mise en œuvre de la méthodologie proposée confirme la richesse des approches que véhicule le concept de transformation tout en proposant l'exploration de la réception humaniste des textes anciens, champ encore relativement minoritaire dans les études de réception.

Alexia Dedieu

Université Grenoble Alpes alexia.dedieu@univ-grenoble-alpes.fr

Jacob BALDE, Jephtias Tragoedia/La Fille de Jephté, tragédie, édition de Dominique Millet-Gérard, Paris, Classiques Garnier, 2020, 668 p., $58 €$ / ISBN 9782406097167.

Longtemps négligée par les historiens du théâtre, la production dramatique des Jésuites en Europe a connu un regain d'intérêt à la faveur des études consacrées à la mouvance baroque. L'importance de ce corpus, signalée dès I954 par Jean Rousset, a été notamment révélée au public francophone grâce aux travaux de JeanMarie Valentin. Toutefois, si l'on dispose désormais d'une documentation nourrie sur la scène scolaire et son environnement, l'accès aux textes reste encore malaisé. C'est pourquoi la vaste et courageuse entreprise de Dominique Millet-Gérard [DMG], qui propose l'édition critique, accompagnée d'une traduction, de la Jephtias de Jacob Balde, mérite d'être saluée avec reconnaissance.

Ce professeur de rhétorique actif en Bavière, que l'on a désigné comme l'Horace germanique en référence à une production poétique aussi abondante que raffinée, se place tout aussi bien dans le sillage de Sénèque pour ce qui est de ses œuvres dramatiques. Jephtias en constitue la réalisation la plus aboutie. Ainsi qu'en témoigne la periocha, programme dans lequel figure le résumé de l'action et le nom des jeunes acteurs, la pièce fut représentée en 1637 à Ingolstadt, dans une première version qu'amplifiera considérablement l'édition tardive publiée à Amberg en 
1654. C'est cette dernière qui sert de base à l'édition très soigneusement établie par DMG, qui en reproduit le texte à l'identique, à la réserve de quelques aménagements de la ponctuation. La langue poétique très travaillée du jésuite alsacien fait que le lecteur, fût-il "suffisant», se reportera volontiers à l'élégante traduction donnée en regard, dont la mise en page se conforme exactement à celle des vers originaux. On appréciera l'option pragmatique d'une transposition soucieuse de lisibilité autant que de fidélité, source d'un texte vivant, ponctué de formules inventives, parfois audacieuses. Loin d'être éludées, les difficultés que peut poser un texte à la complexité parfois redoutable deviennent le tremplin d'hypothèses toujours stimulantes. Outre l'abondante annotation sur laquelle il y aura lieu de revenir, la présentation du texte est enrichie par l'indication marginale des séquences métriques subtilement agencées dans les parties lyriques confiées aux chœurs. Ces repères sont explicités dans une note analytique très savante sur l'utilisation des modèles de versification sénéquiens dans l'œuvre du jésuite.

Comme ses devanciers, parmi lesquels figure l'humaniste écossais Buchanan, Balde s'emploie à réaménager les données de la source biblique (Juges II), dont on sait qu'elle a toujours représenté une énigme, sinon un écueil, aux yeux des exégètes. La calamité du vœu imprudent qui contraint un père à sacrifier sa fille unique appelle nécessairement une démarche herméneutique, dont l'orientation va conditionner l'économie même du drame. Les exigences de la scène moderne invitent par ailleurs à revêtir les protagonistes du récit biblique d'une identité singulière. C'est ce que suggère en particulier le nom que donne Balde à la fille anonyme de Jephté, Menulema, anagramme d'Emmanuel, sur laquelle repose la lecture christique du sacrifice, que privilégie le jésuite. À son héroïne, tendue entre le stoïcisme sénéquien et le sublime de l'interprétation allégorique, Balde associe le personnage fictif d'Ariphanasso, dont le nom est à son tour l'anagramme de Pharaonissa, la Bien-Aimée du Cantique. Ainsi, d'ornement conventionnel, les amours de Menulema et d'Ariphanasso subissent une transfiguration symbolique qui en font l'annonce des noces du Christ et de son Église. Cette modulation du propos correspond bien à la note propre au théâtre jésuite qui, à la vocation pédagogique inaugurée par son modèle humaniste, joint une préoccupation apologétique en accord avec l'élan missionnaire caractéristique de la pastorale post-tridentine.

Au début de l'Acte III, qui se conclura sur le face-à-face terrible du sacrificateur malgré lui et de sa victime, la jeune Menulema apparaît devant son métier à tisser, où elle s'applique à reproduire le tableau d'Isaac sacrifié. La symbolique prémonitoire donne un relief tout particulier à cette scène, par ailleurs assez courante dans le registre de l'épopée chrétienne vouée aux héroïnes bibliques : dans le prolongement de la Judit de Du Bartas, les Suzanne et les Madeleine s'adonneront elles aussi à cette pratique de l'image sur toile, qui trahit peut-être un souci de mitiger leurs destinées exceptionnelles en les maintenant aussi longtemps que possible dans la chambre des dames. En l'occurrence toutefois, la dimension spéculaire de cet épisode ne se borne pas à une anticipation thématique. Il est en effet permis de voir dans le geste artiste de la tisserande la métaphore d'une écriture nouant à l'infini les fils d'une tradition inépuisable. Que la production d'un professeur de rhétorique soit par définition vouée à l'intertextualité relève de l'évidence. Mais la diversité et la densité d'une texture, parfois à la limite du centon, présente l'esthétique «arachnéenne » de Balde comme un espace de fascination continuelle. Ce procédé poussé à sa mesure extrême donne du reste à rêver sur les compétences d'un lectorat en com- 
paraison duquel, comme le rappelle plaisamment DMG, nous sommes devenus des nains. Nous aurons cependant bien garde de confondre dans notre irrémédiable analphabétisme contemporain l'immense culture de celle dont l'accompagnement secourable reflète l'ancienne pédagogie de la praelectio, commentaire soucieux de déployer, dans la lenteur, toutes les composantes cachées d'un texte. L'éditrice se meut avec une érudition confondante dans le vaste corpus des sources où tous les registres lui semblent familiers, de la tradition littéraire et rituelle de l'Antiquité à la Bible et aux réminiscences liturgiques, en passant par la production néo-latine contemporaine, pour ne rien dire des autres compositions de Balde, lequel puise régulièrement dans son propre trésor. À cette somme d'érudition par touches discrètes, on ajoutera quelques échappées rafraîchissantes dans le domaine de la flore ou de la parfumerie exotique. Contre toute attente, l'imposant appareil des notes explicatives qui accompagne la traduction du texte n'est jamais pesant, étant habité en permanence par une curiosité communicative qui revêt de grâce l'exercice du savoir.

L'espace manque pour évoquer comme ils le mériteraient les liminaires et les annexes qui, à la faveur une fois encore des commentaires attentifs qui en éclairent la portée, contribuent à situer la Jephtias en relation avec son milieu, ainsi qu'à préciser les prises de position du dramaturge par rapport aux théories de son temps. La documentation bibliographique impressionnante, qui s'aligne sur les trente dernières pages de cette publication magistrale, reflète la qualité d'un travail de recherche dans lequel nous reconnaissons un exemple lumineux des fruits que dispense une démarche philologique bien comprise.

Simone de REYFF

Université de Fribourg (Suisse) simone.dereyff@unifr.ch
Joshua Billings, Felix Budelmann et Fiona Macintosh (éds.), Choruses, Ancient andModern, Oxford et New York, Oxford University Press, 20I3, 424 p., £I3o /

ISBN 9780199670574.

Le volume collectif Choruses, Ancient \& Modern publié aux presses universitaires d'Oxford et édité par les trois hellénistes Joshua Billings, Felix Budelmann et Fiona Macintosh se propose, en partant du chœur grec, d'explorer à travers une vingtaine de contributions ses métamorphoses depuis l'Antiquité jusqu'à nos jours.

Il s'ouvre sur une introduction solide qui met bien en lumière les enjeux de l'entreprise. Sans se limiter à un type de chœur - même si le chœur tragique est ici prédominant - les auteurs soulignent l'importance des pratiques chorales en Grèce ancienne, dans leur diversité, et rappellent leur rôle dans la construction de la communauté : to take part in a chorus was to be embedded in a social texture and to have a share in the pleasures of community. Cette importance met en lumière à la fois leur moindre présence dans les cultures modernes et le désir récurrent de ces cultures de reconstruire, réinventer quelque chose de similaire - ce que les auteurs qualifient joliment de « nostalgie». En analysant ce rapport moderne au chœur grec grâce à une réflexion transhistorique, ils entendent “ défamiliariser » notre compréhension de ce dernier. Ce sont ainsi les réponses créatives au problème que constitue, pour les modernes, le chœur, qui sont examinées : Schiller, Nietzsche, Brecht sont ainsi convoqués. L'idéalisme allemand et Aristote occupent une part importante de la réflexion, mais les auteurs sont également attentifs à d'autres approches, s'inspirant notamment des sciences cognitives. Ces dernières ont en effet montré qu'être acteur et spectateur d'un chœur activait les mêmes neurones, la différence se mesurant moins en termes de nature de l'expérience que de degrés. 
Le volume comprend quatre parties. La première, Scholarship, revient sur les discours tenus sur le chœur grec à travers les âges. Le premier chapitre, qui s'intéresse aux propos des Anciens sur le chœur est particulièrement intéressant: Anastasia-Erasmia Peponi y analyse les textes de Platon, Aristote et d'autres auteurs et montre l'évolution que connaît la conception du chœur. Les deux chapitres suivants, de Simon Goldhill et Constanze Güthenke, portent sur le “moment» allemand du $\mathrm{xIX}^{\mathrm{e}}$ siècle dont nous sommes encore largement les héritiers, entamé cependant par les travaux en anthropologie sur les pratiques chorales traditionnelles, qui ont amené à reconsidérer le chœur comique sous un nouvel angle, comme le montre Ian Rutherford. Le deuxième partie, Aesthetics, s'intéresse aux contextes artistiques dans lesquels s'inscrivent les pratiques chorales: en Grèce archaïque et classique (Felix Budelmann), dans les tragédies de Sénèque (Hélène Slaney), dans l'opéra (Roger Savage), dans le théâtre européen du xvıI ${ }^{\mathrm{e}}$ siècle (Joshua Billings) et dans celui de Brecht (Martin Revermann). La troisième partie, Shadows, s'intéresse à la nostalgie de la modernité concernant le chœur antique et sa possible absence: Christian Biet analyse ainsi l'absence de chœur dans le théâtre classique français à l'aune des tensions religieuses qui divisent la communauté, Cécile Dudouyt s'intéresse au chœur "fantôme " chez Voltaire, Laurence Dreyfus au chœur chez Wagner comme une forme purement musicale et Zachary Dunbar au chœur dans les comédies musicales de Broadway, dans un contexte social hautement individualiste. Enfin, la dernière partie, Community, est consacrée à la dimension sociale des performances collectives: en Grèce (Richard Seaford), au XvIII ${ }^{\mathrm{e}}$ siècle dans sa dimension politico-sociale (Edith Hall), au xIx $^{\text {e }}$ siècle avec le corps de ballet (Fiona Macintosh), au $\mathrm{xx}^{\mathrm{e}}$ siècle avec le nazisme (Eleftheria Ioannidou), à la fin du $\mathrm{xx}^{\mathrm{e}}$ siècle avec le choric theatre allemand qui fait de la tension entre le collectif et l'individu le centre de ses préoccupations (Erika Fischer-Lichte). Le volume se clôt avec le chapitre d'Helen Eastman sur le théâtre britannique contemporain qui revisite les pratiques chorales.

Par-delà l'ampleur du sujet, qui est loin d'être couvert, et la diversité des contributions, qui sont parfois très éloignées les unes des autres, le volume vaut le détour en raison de la très grande qualité de toutes les contributions, de son introduction magistrale et des pistes de réflexion et d'approfondissement qu'il ouvre.

$$
\begin{array}{r}
\text { Malika Bastin-Haмmou } \\
\text { Université Grenoble-Alpes } \\
\text { malika.bastin@univ-grenoble-alpes.fr }
\end{array}
$$

Tiphaine Annabelle Besnard et Mathieu Scapin (dir.), Age of Classics! L'Antiquité dans la culture pop. Catalogue de l'exposition présentée au Musée SaintRaymond, Musée d'archéologie de Toulouse, du 22 février au 22 septembre 20Ig, Toulouse, Musée Saint-Raymond, 20I9, 2I9 p., 25 €/ ISBN 9782909454436.

Tiphaine Annabelle Besnard et Mathieu Scapin, commissaires de l'exposition Age of Classics! L'Antiquité dans la culture pop, nous proposent un catalogue associé de plus de 200 pages, richement illustré. Le catalogue se compose de sept parties thématiques, d'une explication de la scénographie de l'exposition, d'un cahier d'illustrations et d'une bibliographie sélective. Le lecteur peut ainsi suivre un parcours, de la définition de la réception de l'Antiquité, dans une première introduction de Pascal Payen, jusqu'à sa “mise en scène " au musée. Après la définition de l'objet scientifique Réception, Sabine Forero Mendoza propose une seconde 
introduction sur l'Antiquité au prisme de l'art contemporain, en soulignant combien les formes antiques sont présentes encore aujourd'hui dans l'art, dépassant même les frontières du monde dit occidental.

S'ouvre ensuite la première partie du catalogue, sur l'Antiquité cinématographique. Matthieu Soler commence par traiter le péplum, autour de huit questions permettant d'avoir une bonne idée des thèmes, des périodes et des aires représentées, mais également des producteurs et des publics. L'Antiquité, à la fois “ terrain de jeu » et objet exotique, permet de traiter bien des sujets, grâce à son éloignement dans le temps, qui fonctionne comme une mise à distance. Puis Fabien Bièvre-Perrin propose d'analyser le vidéo-clip à travers quelques exemples particuliers. Il montre combien les références classiques sont partagées, sans être pour autant maîtrisées, et qu'elles sont investies par les artistes et réalisateurs qui proposent ainsi, en la revisitant, une version actualisée de la matière classique.

La deuxième partie du catalogue est consacrée aux jeux vidéo. Romain Vincent commence par remarquer que l'Antiquité se prête très bien - par son réservoir inépuisable de récits, de héros et de créatures - à la ludoformation, autrement dit le processus de transformation du réel en jeu. Autour de cas particuliers, il aborde les thèmes de l'utilisation des savoirs, de l'accès pour le joueur au contenu culturel et du défi de la reconstitution du réel, tout en resituant chaque jeu dans son contexte propre. C'est ensuite Jean-Claude Golvin qui prend la main. Scientifique et auteur de nombreuses restitutions, il nous livre une contribution éclairante sur les méthodes possibles, par ordinateur ou tout simplement à la main, pour reconstituer le passé, en détaillant les enjeux et les problématiques, scientifiques et de transmission aux publics, de chacune.

L'Antiquité mise en bulles est la partie suivante, consacrée à la bande dessinée francophone. Julie Gallego nous offre pour démarrer un panorama efficace des créations depuis Jacques Martin et son célèbre héros Alix. Â partir des années 2000 en particulier, on assiste à une diversification des thèmes, montrant encore une fois que l'Antiquité est une source d'inspiration intarissable pour bien des médiums de la culture populaire. C'est ensuite Valérie Mangin qui retrace son parcours et son rapport à l'Antiquité depuis sa découverte d'Alix jusqu'à sa carrière de scénariste de bandes dessinées. Elle dévoile ainsi au lecteur les questions des auteurs, les libertés volontairement prises avec la véracité historique mais également le rôle du lecteur qui reçoit ces histoires.

Après la bande dessinée, la quatrième partie est consacrée aux comics américains. Danièle André plante le contexte en revenant sur la société américaine au moment de la fondation des États-Unis jusqu'à aujourd'hui: l'Antiquité y est une référence essentielle et forte qui accompagne l'histoire tant politique qu'architecturale et artistique de ce pays. Mathieu Scapin en vient ensuite aux références antiques utilisées dans les comics. Des références explicites comme Hercule ou les dieux du panthéon olympien, les auteurs sont ensuite passés à des éléments plus implicites comme les attributs, les capacités, les histoires, ancrant désormais les super-héros dans une réalité du quotidien nord-américain, mais qui possède toujours de nombreux parallèles avec les mythologies classiques.

La partie suivante s'intéresse au manga. Mathieu Scapin propose un aperçu général, du point de vue d'un lecteur “ occidental » qui introduit utilement la contribution suivante de Sawako Morita et Yasuo Ikuta sur l'utilisation des classiques d'Occident dans les mangas. L'acceptation de plus en plus grande de la culture classique au Japon ces dernières années a permis de renouveler les thèmes et les approches, même si certains thèmes restent privilégiés, 
comme la mythologie grecque, Alexandre le Grand et les guerres puniques. L'Antiquité entre en dialogue avec la culture nippone dans ces mangas, sans le manichéisme des comics.

Vient ensuite la littérature populaire, présentée par Catherine Psilakis. Elle analyse l'utilisation de l'Antiquité dans des romans à succès pendant trois périodes, la fin du XIx ${ }^{\text {e }}$ siècle, I9oo-ig5o et de I95o à nos jours. Alors que les études classiques sont actuellement moins prisées, l'Antiquité semble au contraire d'une grande vivacité dans ce type de littérature. Sandya Sistac présente ensuite quelques exemples de la littérature dite de jeunesse et y montre les usages qui y sont faits de l'Antiquité, en particulier l'usage des langues anciennes pour penser la différence, et les ressorts de la narration mettant en lumière le rôle du destin comme pour les héros antiques.

La dernière partie aborde la présence de l'Antiquité dans l'art contemporain. Tiphaine Annabelle Besnard propose une réflexion sur la pratique hyperartistique, ainsi que les notions de kitsch, d'inauthentique et de pop. Elle démontre ainsi que l'Antiquité est moderne, au gré des modifications qu'elle subit sur les plans formels et sémantiques dans les œuvres. Dans une deuxième contribution, l'auteure revient sur le but de l'exposition : montrer l'Antiquité dans l'art - ce que font davantage les galeries ou les fondations habituellement, pas les musées - et participer à la visibilité des artistes néo néo qui utilisent la réminiscence ou le travestissement des références classiques dans leurs œuvres. La partie se conclut avec les entrevues de Léo Caillard et de Pierre et Gilles dont plusieurs œuvres sont exposées. Les artistes livrent ainsi leur point de vue sur leur propre utilisation de la matière antique. Vient enfin une entrevue du designer de l'exposition, Guillaume Lamarque, qui explique la scénographie de l'exposition.
L'ouvrage est réussi et original, à la fois ludique et agréable, et solide sur le plan scientifique. L'ensemble des contributions, claires et concises, présente une bibliographie représentative du thème. Surtout, il s'agit ici d'un vrai travail de réception de l'Antiquité qui doit être salué, avec une attention identique portée à l'objet Antiquité et aux supports qui l'accueillent, la transforment et au final continuent de la faire vivre encore aujourd'hui.

$$
\begin{array}{r}
\text { Élodie GUILLON } \\
\text { Université Toulouse-Jean Jaurès } \\
\text { elodie.guillon@univ-tlse2.fr }
\end{array}
$$

Fiona Cox, Ovid's Presence in

Contemporary Women's Writing. Strange

Monsters, Oxford et New York, Oxford

University Press, 20I8, 272 p., £6o, ISBN 9780198779889.

L'importance des travaux de Fiona Cox dans le domaine de la réception de l'Antiquité et des gender studies n'est plus à démontrer. La chercheuse a en effet confirmé comme son principal objet d'étude les (ré-)écritures féminines d'auteurs anciens majeurs, de Virgile (Sibylline Sisters. Virgil's Presence in Contemporary Women's Writing, Oxford University Press, 20II) à Homère (Homer's Daughters. Women's Responses to Homer in the Twentieth Century andBeyond, avec Elena Theodorakopoulos, Oxford University Press, 20I9) en passant ici par Ovide. Ce dernier, dont le xxi ${ }^{\mathrm{e}}$ s. semble pouvoir constituer l'aetas - aetas Ovidiana - non moins que le $\mathrm{xx}^{\mathrm{e}}$, a fait l'objet de nombreuses appropriations de la part de femmes auteures ; F. Cox souligne dans son introduction que celles-ci n'ont pas encore été étudiées de manière systématique, un manque auquel elle a donc choisi de remédier.

Cette introduction nous plonge tout de suite dans le vif du sujet: elle contient 
certes une mise au point sur le contexte particulier dont relèvent les auteures sur lesquelles elle a choisi de travailler - celui de la troisième vague féministe, dans laquelle le discours des femmes s'empare de questionnements politiques et sociaux et s'emploie à inclure les moins favorisé.e.s -, mais elle comporte aussi déjà un certain nombre d'analyses de textes et de multiples références. Le propos n'est pas toujours aisé à suivre et il est parfois allusif de sorte qu'un lecteur non francophone ou non familier des œuvres citées peut avoir un peu de peine à suivre et à entrer ainsi dans le livre. La deuxième partie de l'introduction est occupée par la présentation d'une nouvelle d'A. S. Byatt, “The Stone Woman » parce qu'elle recèle la plupart des thèmes que vont ensuite illustrer les œuvres des auteures considérées dans les onze chapitres de l'ouvrage : la métamorphose relie la plupart de ces thèmes (la maladie, l'exil, l'horreur des guerres, le capitalisme et la cupidité, les conséquences de politiques de santé restrictives, internet et l'e-réputation, le réchauffement climatique...), ainsi que l'idée qui apparaît dans le sous-titre du livre, “Strange Monsters » (originellement, une citation de May Sarton, comme il est rappelé dès la p.4). Un thème majeur qu'illustre la nouvelle citée d'A. S. Byatt est “ le pouvoir que la perspective et le contexte ont de transfigurer notre notion de "monstrueux" en notion de "merveilleux" » (p. 24). L'organisation de l'ouvrage soulève ainsi quelques questions (outre le traitement d'A. S. Byatt dans l'introduction, c'est aussi le regroupement de deux auteures dans les chapitres 9 et Io), mais sa cohérence d'ensemble demeure indiscutable, les transitions entre les chapitres sont soignées, les points communs entre les auteures sont régulièrement relevés et le dernier chapitre, consacré à Jane Alison, commence lui-même par une utile synthèse, avant qu'une vraie conclusion ne réunisse les nombreux fils déroulés par l'ouvrage.
Le moins qu'on puisse dire de cet ouvrage est qu'il montre à quel point le carmen perpetuum d'Ovide - compris comme un chant qui ne s'arrête jamais et ne s'est jamais arrêté - est encore vif et actuel et avec une variété remarquable d'interprétations. Et il ne s'agit pas que des Métamorphoses auxquelles l'expression carmen perpetuum appartient et qui se concluent, comme on le sait, sur le vœu du poète de rester vivant par son œuvre. Un apport conséquent de l'ouvrage concerne en effet les multiples entreprises de réélaboration, voire de transformation, que les élégies de l'exil, Tristes et Pontiques, ont suscitées de la part des auteures: pour reprendre une image développée par Marie Darrieussecq dans la préface à ses Tristes pontiques, l'appel qu'Ovide paraît nous lancer depuis le « Pont triste » a véritablement été entendu par ces écrivaines ; il a même donné lieu à une réponse qui, pour reprendre, cette fois, une référence que F. Cox reprend à Ali Smith (voir p.46) et mobilise souvent tout au long du livre, n'est pas un simple écho à la façon d'Écho dans les Métamorphoses : c'est une vraie réponse en retour qui engage des sujets aussi bien personnels que publics et collectifs (le meilleur exemple est sans doute le poème The word for sorrow de Joséphine Balmer - présenté dans la première partie du chapitre 9 - qui fait se rencontrer l'expérience de l'exil d'Ovide et celle de soldats de la Première Guerre mondiale postés à Gallipoli en Turquie et qui mène aussi l'auteure à revenir sur sa propre expérience de la perte d'un être cher).

La grande réussite de l'ouvrage de F. Cox est, certainement, de nous faire mesurer tout le dynamisme créatif et politique du féminisme de la troisième vague non moins que de nous faire réfléchir, à peu près pour chaque auteure considérée, sur les relais qui jalonnent la réception d'Ovide en général et cette réception d'Ovide en particulier (avec des œuvres et des auteur.e.s récurrent.e.s comme la Tempête de Shakespeare, 
E. Dickinson, T. S. Eliot, V. Woolf...). Mais, surtout auprès du public non anglophone que j'évoquais précédemment, sa réussite tient aussi en sa capacité à nous donner envie de découvrir, de lire (ou mieux lire) les différentes œuvres qu'elle aborde et qu'elle décrit avec finesse. Pour y inviter d'autres lecteurs, je reprendrai pour finir les titres des principales œuvres: Public Library et Autumn d'Ali Smith (ch. I) ; The Leto Bundle de Marina Warner (ch. 2); Opium für Ovid d'Yoko Tawada (ch. 3); Dart d'Alice Oswald (ch.4); Metamorphoses: A Play de Mary Zimmermann (ch.5); Google me! et For a Barbarian Woman de Saviana Stanescu (ch.6); Of Mutability de Jo Shapcott (ch. 7); Tristes pontiques mais aussi White et Tom est mort de Marie Darrieussecq (ch. 8); The Word for Sorrow de Josephine Balmer et Song and Error d'Averill Curdy (ch. 9), la traduction des Hérö̈des par Clare Pollard (ch. Io), les traductions de J. Alison ainsi qu'un livre de mémoires, The Sisters Antipodes (ch. II).

Séverine Tarantino

Université de Lille severine.tarantino@univ-lille.fr

\begin{abstract}
Annick Fenet, Michela Passini et Sara
Nardi-Combescure (dir.), Hommes

et patrimoines en guerre. L'heure

du choix (IgI4-IgI8), Dijon, Éditions

universitaires de Dijon, 2018, 302 p., $20 € /$

IBSN 9782364/42538.
\end{abstract}

L'ouvrage édité en 2018 par Annick Fenet, Michela Passini et Sara Nardi-Combescure, Hommes et patrimoines en guerre. L'heure $d u$ choix (IgI4-IgI8) prend place parmi les abondants travaux historiques, qui ont paru avec le centenaire de la Première Guerre, et le petit nombre d'entre eux qui s'est intéressé aux questions patrimoniales et culturelles liées au conflit. L'originalité de ces actes d'un colloque de 20I7 est d'associer les différentes périodes artistiques, de l'Antiquité à l'art du $\mathrm{xIx}^{\mathrm{e}}$ siècle, et de décentrer et déseuropéaniser le regard, en ouvrant la focale depuis le patrimoine européen (sont abordés des dossiers français, britannique, allemand, italien, belge, espagnol) vers l'Est, le Moyen Orient et l'Asie centrale (Syrie, Anatolie, Turkestan).

Histoire transnationale et culturelle. Ce qui permet cette ampleur de vue chronologique et géographique, ce sont d'une part les spécialités respectives des trois éditrices (pour l'archéologie asiatique mentionnons en particulier les travaux d'A. Fenet); et il faut saluer qu'une telle entreprise ait vu le jour en France, où l'histoire del'histoire del'art et en particulier la réception de l'Antiquité ne réussissent pas encore à prendre leur place au sein des disciplines universitaires. D'autre part, la très intéressante introduction d'A. Fenet et M.Passini montre tout ce que cette orientation doit aux recherches menées en histoire transnationale, telle qu'elle a été pratiquée par exemple dans le programme TransferS, auquel est rattachée la publication. Une autre orientation historiographique à laquelle se réfèrent les éditrices du volume est l'histoire culturelle de la Grande Guerre. Mettant l'accent sur les individus qui ont vécu le conflit à travers leurs affects, leurs imaginaires et leurs représentations, elle se différencie d'une histoire sociale, plus attentive aux chaînes de commandement et de domination, aux contraintes sociales et économiques, plus alertée par les biais d'une documentation produite par une même classe sociale. Les acteurs auxquels s'intéressent les treize contributions de cet ouvrage sont, il est vrai, issus de la traditionnelle aristocratie et bourgeoisie qui fournissent encore, au début du $\mathrm{xx}^{\mathrm{e}}$ siècle, les seuls professionnels du patrimoine. Repris par le plan de l'ouvrage, de manière plus ou moins cloisonnée, trois objectifs émergent du projet scientifique de l'entreprise. 
La fabrique d'une histoire intellectuelle. Reprenant une démarche mise en œuvre dans un ouvrage récent del'une des éditrices (M. Passini, La fabrique de l'art national, 2012), on peut apprécier la diversité des travaux effectués en temps de guerre par les intellectuels étudiés dans les contributions, afin de construire cette histoire culturelle à travers les pratiques. Certains sont sur le front, comme les archéologues italiens (M. Munzi), dont les carrières, en "sortie de guerre », en profiteront. D'autres restent sur les arrières, faisant avancer la science. Les travaux de collaboration internationale sont dans l'ensemble suspendus (È. Gran Aymerich), mais les historiens continuent à écrire (exemple de Franz Cumont en Belgique étudié par C. Bonnet), à éditer (exemple de Salomon Reinach faisant paraître la Revue Archéologique dans l'étude de H. Duchêne), à faire cours (exemples de la Sorbonne, du Collège de France, de l'EPHE, de l'École du Louvre et des chaires britanniques d'Art History, dans les textes d'A. Fenet, J. von Ungern-Sternberg et É. Oléron Evans). Ces hommes prennent en charge la protection des collections (F.Pagano), ainsi que leur inventaire (exemple du réseau allemand de Paul Clemen pour le patrimoine occupé, dans la contribution de C. Kott). Et même, dans certaines régions, ils continuent à fouiller : d'importantes mises au jour archéologiques en Italie (M. Munzi) éclipsent les découvertes du lointain Turkestan (S. Gorshenina). Le contexte de tension internationale semble aussi avoir favorisé des découvertes, comme le déchiffrement à Vienne et à Berlin du hittite en tant que langue indo-européenne (S. Alaura).

Une époque charnière. Si le conflit marque historiquement un tournant du $\mathrm{xIx}^{\mathrm{e}}$ siècle vers le monde contemporain, plusieurs évolutions, dans les pratiques étudiées ici ainsi que dans les théories de l'archéologie et de l'histoire de l'art, sont pointées dans l'ouvrage. Le premier signe d'une mutation réside dans l'adoption du terme
“ patrimoine », qui, comme l'indique l'introduction, était peu utilisé avant la Première Guerre. On mentionnera deux autres transformations. Les arts anciens constituaient encore le fondement de la discipline, comme l'illustre la majorité des exemples traités. Toutefois ce modèle commence à céder la place aux périodes chronologiques plus récentes, tandis que se construit la discipline d'une histoire continue de l'art, illustrée par la Grande-Bretagne (É. Oléron Evans). Le second apport est l'évolution que connaissent les musées européens, qui prennent alors conscience de l'importance de la sauvegarde de la mémoire collective et du patrimoine (F. Pagano), revoient leur politique de documentation des collections ainsi que leurs missions d'enseignement, en s'affranchissant du modèle allemand (rôle des visites-conférences au Louvre, repris dans les pays anglo-saxons : M. Tchernia-Blanchard).

Une histoire de l'engagement. Un dernier atout du volume est l'attention portée aux hommes eux-mêmes et à leur engagement vis-à-vis du conflit. Cette thématique n'est possible qu'avec la prise en compte des différents contextes politiques nationaux (l'histoire du Turkestan au sein des révolutions soviétiques brossée par S. Gorshenina; les liens entre Risorgimento, Grande Guerre et fascisme rappelés par M. Munzi ; la germanophilie en Espagne dans l'étude de F. Gracia-Alonso) et elle tire sa dynamique de l'illustration équilibrée des deux camps : les vainqueurs et les vaincus. La présentation d'une série de portraits, plus ou moins rapides, de chercheurs permet ainsi d'aborder des questions d'idéologie et de propagande politiques, dans lesquelles les arts servent de munitions. Ainsi, dans cette guerre scientifique, modèle grec et romain s'opposent, tandis que le bombardement de la cathédrale de Reims en igı/ est comparé à l'explosion du Parthénon en i687.

C'est en mobilisant des sources inédites et variées que l'équipe internationale, 
réunie autour de ce livre, compose donc une vision complète et passionnante de cette période historique.

Natacha LUBTCHANSKY

Université de Tours lubtchansky@univ-tours.fr

Kristopher F. B. Fletcher et Osman UmurHan (éds.), Classical Antiquity in Heavy Metal Music, Londres et New York, Bloomsbury Academic, 2019, 26o p., £90 / ISBN 978I35007535ı.

Nell'Introduzione di questo volume collettivo intitolata "Where Metal and Classics Meet", gli editori, Fletcher et Umurhan, fanno partire la loro analisi da un'interessante riflessione: questo libro è stato concepito non tanto per l'incredibile quantità di brani e album di musica metal facenti riferimento o basati sull'antichità classica. Piuttosto li ha colpito come ci sono pochi corsi di storia antica che non sarebbero degnamente accompagnati da una lunga e ricca playlist di brani metal ispirati ai fatti snocciolati nell'arco dei suddetti corsi. Tra storia greca e storia romana, passando dall'Egitto e l'Europa celtica, gli esempi di brani ispirati ad opere, fatti o figure caratteristiche di quel periodo sono numerosissimi. Di conseguenza era decisamente ora che qualcuno si impegnasse per improntare un'analisi e un confronto tra storia antica e musica moderna di stampo metal.

Per centrare questo obiettivo, gli editori hanno raccolto in un volume della ricca e stimolante collana "Imagines" nove saggi, di cui due scritti proprio da ciascun editore. $\mathrm{Al}$ di là del rendere noto al pubblico accademico e non di questo stretto e pregnante nesso fra antichità e musica metal, il merito dei vari autori sta soprattutto nell'analizzare le modalità di ricezione dell'antichità nei testi e nelle atmosfere di queste opere musicali. Infatti si evince che ognuna di queste opere si approccia a tematiche prettamente storiche da un punto di vista e con una lente diversa. Di fondamentale importanza risulta anche l'analisi del contesto storico in cui questa musica è stata prodotta, in diversi paesi, come l'Italia, la Grecia, per diversi pubblici, con vari intenti.

Ad esempio, le parole degli Stormlord, band italiana, ci mostrano il lato "umano" di Enea e del suo amore per Didone, la difficoltà delle scelte da lui intraprese nel suo cammino verso la "fondazione" della nazione italiana. I greci Kawir reinterpretano e fanno propri inni cultuali del pantheon greco antico, modernizzandoli e dando loro nuova vita nella musica black metal. Allo stesso modo gli americani Nile fanno abbondante uso della storia dell'Egitto faraonico nel loro death metal estremo per trasmettere all'uditorio immagini e sensazioni forti, definite "antropologicamente trasgressive" dagli autori.

Il primo capitolo analizza la ricezione dell'Eneide di Virgilio nella musica metal, e in particolare nelle opere delle band italiane Stormlord, Heimdall e Hesperia. Nello specifico viene analizzato il ruolo del nazionalismo e dell'identità nazionale, e il suo rapporto con l'antichità nel heavy metal italiano. Nel secondo capitolo, si vede come l'appropriarsi della storia romana e il conflitto con i popoli celtici, contribuisce alla ricostruzione di una sorta di orgoglio celtico attraverso i versi della musica di band come gli Eluveitie o Suidakra. Il terzo capitolo è dedicato alla modernizzazione e reinterpretazione di inni rituali greci come marchio di fabbrica di alcuni gruppi greci attuali. La visione della musica metal come tipicamente ispirata all'identità maschile è oggetto del quarto capitolo, dove gli autori scelgono di superare questo concetto e di proporre un approccio ispirato ai gender studies . Nello specifico analizzando generi come il gothic metal e power metal, generi 
in un certo senso opposti, si vede come essi abbiano rielaborato in modo creativo figure storiche femminili come Cassandra o Poppea. Seguendo tematicamente il capitolo precedente, nel quinto viene analizzata la ricezione e "traduzione" in musica del mito di Didone e Enea, e della forte personalità della regina di Cartagine in opposizione alla tradizionale forte mascolinità presente nella musica metal. Il sesto capitolo tratta la percezione della figura di Caligola nell'album della band Ex Deo a lui dedicato, e esplora il ruolo del male e della cattiveria brutale all'interno della musica metal, da sempre tematica maggiore. Il settimo capitolo si incentra sulle tematiche esoteriche e arcane come vengono ritrovate nell'opera della band Ancient Rites. Nei suoi brani si ritrova un'antichità greca e romana vista attraverso la lente dell'esoterismo, che già di per sé è cara alla musica metal, trasmettendone così aspetti particolari e molto personali. L'ottavo capitolo tratta il tema dell'Antico Egitto e la sua ricezione all'interno della musica metal, con un focus sul lavoro degli americani Nile, che hanno ampiamente sfruttato queste tematiche. Nonostante ciò, notano gli autori, gli storici dell'Antico Egitto hanno del tutto ignorato questa forma particolare di ricezione. Il nono e ultimo capitolo raccoglie le mode e i trend prominenti, rintracciati dagli autori nella ricezione dell'antichità in seno alla musica metal moderna.

Che si tratti di Cassandra o di Caligola, dell'Egitto, della Grecia o di Roma, l'esplorazione dei processi creativi ispirati all'Antichità sotto una lente al contempo storica, filologica e antropologica risulta molto interessante. Il superamento dei pregiudizi e dello stigma sociale spesso associato al genere ed ai fruitori della musica heavy metal permette una lucida analisi di contenuti originali, radicati in un immaginario collettivo. Il libro va salutato come un apporto significativo allo studio della ricezione dell'antico nell cultura "pop" (cioè popolare : fumetti, musica, video...).

Francesco XeLLA

Rome

francescoxella@hotmail.com

Corinne BonNET

Université Toulouse - Jean Jaurès cbonnet@univ-tlse2.fr

Shawn W. Fuynn (éd.), Children in the

Bible and the Ancient World. Comparative and HistoricalMethods in Reading

Ancient Children, Londres, Routledge, 20I9, 226 p., £96 / ISBN 978II38543768.

L'enfance a trouvé sa place dans les études classiques depuis une trentaine d'années. Ce thème est en revanche entré plus récemment dans le champ de l'histoire de l'Orient ancien, et notamment des mondes de la Bible. Les publications relatives à l'enfance et aux enfants se sont multipliées ces dernières années. Nous pouvons notamment citer les ouvrages de K. Garroway, Growing up in Ancient Israel (2019), D. Bosworth, Infant weeping in Akkadian, Hebrew and Greek Literature (20I6) ou H. Dewrell, Child Sacrifice in Ancient Israel (20I7). C'est donc sans surprise que nous retrouvons plusieurs articles de ces spécialistes dans l'ouvrage dirigé par S. Flynn, lui-même auteur de Children in Ancient Israel. The Hebrew Bible and Mesopotamia in Comparative Perspective (2018).

La première partie de l'ouvrage est consacrée au monde de la Bible et, plus largement, au Proche-Orient ancien. H. Dewrell interroge la place que les enfants occupent dans les vœux adressés à la divinité en comparant la bible hébraïque aux sources ougaritiques. Il montre que le souci de s'assurer une descendance y occupe une place importante, mais que les enfants eux- 
mêmes peuvent être offerts à la divinité, qu'il s'agisse du sacrifice du premier-né ou de la consécration d'un enfant à la divinité (p. 3-ı6). C. Bergmann propose une reconstitution des connaissances obstétricales que pouvaient avoir les auteurs de la Bible hébraïque à travers les récits d'accouchements dystociques, dont la portée était toutefois avant tout métaphorique. L'autrice interroge également la dimension théologique de ces récits de naissance, notamment autour du motif de la délivrance: celle de l'enfant au moment de l'accouchement renverrait par exemple à la sortie d'Égypte (p. 17-34). D. Bosworth s'intéresse aux abandons d'enfants dans l'Ancien Testament et dans la littérature grecque par le biais d'une démarche comparatiste. La démonstration repose essentiellement sur des données littéraires : les abandons de Moïse et d'Ismaël sont ainsi comparés aux récits mythologiques d'expositions de nouveau-nés dans les sources d'époque romaine (p. 35-58). K. Garroway s'intéresse à la place des jeunes filles nubiles dans les sociétés proche-orientales, toujours dans une perspective comparatiste, en confrontant les récits bibliques relatifs au mariage ou à l'héritage de filles aux sources mésopotamiennes relatives au droit et à la mythologie. Cette étude met ainsi en lumière le regard porté par un monde d'hommes sur ces jeunes filles ainsi que l'agentivité de ces dernières qui, par conséquent, peuvent mettre en danger l'ordre établi tout en demeurant précieuses pour leur famille, et plus largement pour leur nation. L'autrice adopte une approche renouvelée de l'enfant, dans le but de redonner une voix à ces individus réduits au silence dans nos sources anciennes, composées, pour l'essentiel, par des hommes adultes (p. 59-74).

La deuxième partie traite du monde grécoromain et chrétien. S. Betsworth y propose une lecture comparative du récit de la mise à mort de Jean-Baptiste dans l'Évangile de Marc, et le culte de Déméter et de Perséphone dans le monde gréco-romain.
L'autrice justifie cette mise en perspective par la présence d'éléments culturels et littéraires gréco-romains dans le Nouveau Testament: selon elle, Hérodiade et sa fille renvoient à Déméter et Perséphone, de même que nombre d'éléments, dont la danse, évoquent les Mystères éleusiniens (p. 77-93). J. Martens s'intéresse à la place des enfants dans les Églises fondées par Paul de Tarse et dans ses écrits, notamment en lien avec les questions de la circoncision et du baptême comme rites d'intégration à la communauté. Son étude met elle aussi en lumière la présence de références grécoromaines dans le Nouveau Testament (p. 94-II4). C. Laes consacre son article aux abus sexuels dont les enfants pouvaient être victimes dans le monde romain, en particulier dans le cadre des écoles. L'auteur rassemble ainsi plusieurs sources anciennes qui évoquent la promiscuité entre les élèves ou avec l'enseignant tout en rappelant que les questions de sexualité doivent être envisagées dans leur contexte historique (p. II5-I33).

La troisième partie est consacrée à la culture matérielle enfantine. J.Parker revient sur le dossier des figurines-piliers judéennes datées pour l'essentiel des $\mathrm{VIII}^{\mathrm{e}}-\mathrm{VII}^{\mathrm{e}}$ siècles avant notre ère. L'autrice propose, au gré d'une démonstration convaincante, d'y reconnaître des objets qui ont pu servir de jouets, entre autres usages (p. I37-I49). S. Sheridan consacre son article aux données bio-archéologiques relatives aux enfants inhumés entre le $\mathrm{v}^{\mathrm{e}}$ et le viI ${ }^{\mathrm{e}}$ siècle de notre ère dans l'enceinte du monastère Saint-Étienne à Jérusalem. L'autrice interroge également les liens entre ces petits défunts et les moines. Cette analyse est toutefois rendue difficile par l'absence de sources écrites relatives à leur présence au sein du monastère (p. I5o-I94). L'ouvrage s'achève sur la mise en perspective par D. Kieser d'un texte apocryphe relatif à la vie de Marie et de la situation des adolescentes $\mathrm{du} \mathrm{xxI}^{\mathrm{e}}$ siècle de notre ère. En effet, le Protévangile de 
Jacques traite de la naissance et de l'enfance de Marie, consacrée dans le Temple à l'âge de trois ans, mais qui dut le quitter à douze ans, au moment de la puberté. L'autrice compare les impératifs de pureté rituelle relatifs aux menstruations et de régulation des comportements sexuels des jeunes filles qui y sont suggérés à ceux auxquelles les adolescentes occidentales sont confrontées de nos jours (p. 197-2i6).

Malgré une aire géographique et une chronologie vastes, l'ouvrage fait montre d'une grande unité autour de la méthode adoptée et de la nature des sources privilégiées, en l'occurrence la Bible hébraïque et le Nouveau Testament. Ce recueil d'articles s'inscrit en outre dans une perspective comparatiste avec les sociétés des régions adjacentes, mondes cunéiforme et gréco-romain, tout en mettant en lumière l'agentivité des enfants au sein de ces sociétés.

Sophie Laribi GLaudel Université de Lorraine sophie.laribi-glaudel@univ-lorraine.fr

\section{Erminio Fonzo, Il mondo antico negli scritti di Antonio Gramsci, Mercato S. Severino, Edizioni Paguro, 20I9, I34 p., ı $€ /$ ISBN 9788899509620 .}

Ces dernières années ont vu un nouvel intérêt pour la pensée d'Antonio Gramsci (I89I-I937) dans les études sur l'Antiquité et sa réception. Après un colloque tenu en 2017 à Newcastle sur Antonio Gramsci and the Ancient World, dont les actes sont en cours de publication, et la fondation en 2018 d'un Gramsci Research Network dédié à ce thème, un ouvrage étudiant la relation du théoricien marxiste italien avec l'Antiquité est paru en 20ig. Son auteur, Erminio Fonzo, chercheur en histoire contemporaine à l'Université de Salerne, a analysé les écrits de Gramsci, en se focalisant notamment sur ses très influents Quaderni del carcere - écrits entre I929 et I935, pendant son emprisonnement sous le régime fasciste, et publiés dans l'après-guerre - afin de retracer comment son originale réflexion marxiste s'est confrontée avec l'histoire ancienne.

D'après un marxisme influencé par l'historicisme idéaliste de Benedetto Croce, la connaissance historique - en tant qu'histoire économique et sociale, ainsi qu'histoire des idées - est considérée par Gramsci comme le savoir laïc qui peut le mieux donner les moyens de compréhension du présent et d'élaboration d'une action politique socialiste (p. 23-26). Le passé est donc étudié à partir des urgences politiques du xx siècle : à son sens, comme pour Benedetto Croce, "l'histoire est toujours histoire contemporaine »(p. 125). Pendant ses études au lycée classique de Cagliari (I9o8-І9II) notamment, et ensuite à l'université de Turin (I9II-I9I5), Gramsci reçoit une formation littéraire, linguistique et antiquisante. Durant la Première Guerre mondiale, en suivant la ligne politique pacifiste du Parti socialiste italien, il interprète la culture classique selon une inspiration antinationaliste et antiraciste (p. I6, I9-20, 33-35), politiquement opposée aux mythes nationalistes de la romanità et de la latinité promus par la droite italienne et par Mussolini. Son approche nonidentitaire de l'Antiquité continue pendant son emprisonnement, dans ses notes contre les interprétations fascistes de la langue étrusque (p. 47-49), de Jules César (p. 63-66), d'Homère (p. II4-II5), de l'héritage de Rome pour l'Italie moderne (p. 87- 9i), ainsi que dans le cadre de sa théorisation marxiste du nouveau type d'intellectuel nazionale-popolare au service des classes subalternes: Gramsci imagine la future perte d'importance du latin et du grec dans l'instruction supérieure en Italie, en faveur d'une majeure adhésion pédagogique au développement économique et technique moderne (p. 37-43). 
En même temps, pour le théoricien marxiste, l'Antiquité est un champ privilégié d'analyse historique. En particulier, Gramsci retrace dans l'histoire de la langue et de la littérature latine les origines du caractère “cosmopolite» des intellectuels laïcs et catholiques en Italie, de leur distance des classes populaires, et par conséquent de l'absence d'une culture nazionalepopolare dans l'Italie moderne (p. 5i-73, 9o-96). À la différence de la littérature latine, dont l'originalité et l'autonomie par rapport à la Grèce sont revendiquées dans l'Italie fasciste, le théâtre grec classique est considéré par Gramsci comme modèle positif d'art nazionale-popolare, produit par des grands artistes et accessible au peuple (p. II3-II/4). Dans le même sens, le dirigeant communiste emprisonné voit en Jules César non pas le héros fondateur d'un empire « italien », mais au contraire l'auteur d'une politique de "dénationalisation» de l'Italie ancienne et de ses intellectuels (p. 5I-55, 63-73). Cette interprétation antifasciste de Jules César conduit également à une réflexion sur la notion politique contemporaine de "césarisme », à travers laquelle Gramsci interprète les dictatures du xIx et du xx ${ }^{\mathrm{e}}$ siècles (p. 75-79). Le dirigeant communiste emprisonné réfléchit en outre au rôle des luttes des classes, des invasions "barbares», de la langue latine et de la religion chrétienne dans l'histoire de l'Antiquité tardive (p. 8I-85, 9I-96). Pour ce qui concerne l'histoire del'économie, entant que discours privilégié de l'interprétation marxiste de l'histoire, Gramsci se range du côté des “ primitivistes » qui (comme Marx, Kautsky et, en Italie, Giuseppe Salvioli) soutiennent l'inexistence d'une économie de type capitaliste dans l'Antiquité, contrairement aux “ modernistes » (Mommsen et Rostovtzeff, et les italiens Corrado Barbagallo et Ettore Ciccotti) qui soulignent la dimension capitaliste de l'économie ancienne. D'après son inspiration marxiste, le dirigeant emprisonné voit ainsi dans l'esclavage la caractéristique structurelle de l'économie et de la société dans l'Antiquité, au contraire du capitalisme contemporain (p. 97-II2).

Erminio Fonzo dédie enfin un dernier chapitre aux œuvres consultées par Gramsci. Dans ces dernières pages, et ailleurs dans son ouvrage, on trouve des informations sur les sources historiographiques de Gramsci sur l'Antiquité : notamment, Guglielmo Ferrero, Corrado Barbagallo, Concetto Marchesi, Ettore Ciccotti, Giuseppe Salvioli (p. 20-22, 28-3o, 93, 99-II2, I2I-I22). Ces précieuses indications peuvent aider à reprendre la recherche de l'influence du marxisme dans les études sur l'Antiquité dans l'Italie préfasciste, après les premiers débats en ce sens des années i97o. Il reste ensuite à retracer l'influence posthume de Gramsci sur les antiquisants après la fin du fascisme, en Italie et ailleurs. Plus largement, si on dispose déjà de nombreuses études sur les utilisations fascistes de l'Antiquité, on aurait besoin de nouvelles histoires des réceptions marxistes. Dans cette perspective, on espère que le très utile ouvrage d'Erminio Fonzo puisse représenter le début d'une série de recherches de la part des antiquisants ainsi que des historiens des idées et du marxisme.

Andrea Avalli Università degli Studi di Genova Université de Picardie Jules Verne xba@hotmail.it

Patrick Gray, Shakespeare and the Fall of
the Roman Republic. Selfhood, Stoicism
and Civil War, Edimbourg, Edinburgh
University Press, 20I9, 3o8 p., £8o / ISBN
978I4744127456.

L'ouvrage est la publication de la thèse de P. Gray.Siletitrelaisse penseràuneréflexion sur la fin de la République et les guerres civiles à travers l'œuvre shakespearienne, 
le sous-titre précise davantage le propos. L'enjeu principal est philosophique et porte tout particulièrement sur la conception de l'individualité des protagonistes. Cette dangereuse construction de soi entraîne généralement leur perte. Shakespeare concevait effectivement la fin de la République comme une tragédie. L'auteur expose clairement la position shakespearienne selon laquelle la poursuite de l'invulnérabilité individuelle serait la principale cause de la chute de la République.

La conséquente et solide introduction (p. I-46) replace l'étude des pièces Julius Caesar et Antony and Cleopatra dans une approche comparatiste et pluridisciplinaire qui caractérise l'ensemble de l'ouvrage. En effet, P. Gray utilise un vaste spectre philosophique, allant de l'Antiquité grecque au temps présent, et mobilise des sources classiques et les travaux d'historiens, penseurs, et théologiens, de toutes périodes. Selon l'auteur, Julius Caesar montrerait la République mourante, tandis qu'Antony and Cleopatra concernerait le début de l'Empire. Cette transition pourrait être rapprochée de la crise de l'aristocratie anglaise contemporaine de Shakespeare (p. I77). Les pièces seraient des miroirs pour la noblesse anglaise. Même si l'analyse se focalise sur ces deux pièces, P. Gray n'hésite pas à mentionner souvent Coriolanus.

Deux parties équilibrées traitent chacune d'une pièce: d'abord de Julius Caesar, puis d'Antony and Cleopatra. Chaque partie dispose de deux chapitres et d'une conclusion. Les protagonistes montrent la volonté de transcender les limites humaines dans un contexte exacerbé de violence et de guerres civiles, sans pour autant atteindre une pleine souveraineté. L'individualité est étudiée à travers de multiples composantes, comme l'imperfection humaine, les passions... La différenciation exacerbée des personnages suscite des réflexions multiples, comme les manifestations du néostoïcisme dans les attitudes de protagonistes défaits, comme Brutus ou la quête insatiable du pouvoir. En outre, l'attitude outrancière d'Antoine prisonnier de ses passions rappelle l'ethos médiéval de la licence aristocratique et le monde contemporain du théâtre, selon P. Gray.

Le premier chapitre "A beast without a heart": pietas and pity in Julius Caesar(p.49-94) traite notamment du personnage central du stoïcien Brutus érigé en incarnation du sapiens, une synthèse entre le philosophe Socrate et l'homme d'Etat Caton. Le contexte de la résurgence du néostoïcisme $\mathrm{au} \mathrm{xvI}^{\mathrm{e}}$ siècle doit être pris en compte, notamment pour la caractérisation de la pitié et l'idéal de pietas. L'analyse de Porcia permet notamment de concevoir si la constance est seulement liée à la masculinité. L'étude menée dans le premier chapitre prépare également la discussion menée dans le chapitre suivant ("The Northern star": constancy and passibility in Julius Caesar, p. 95-I44) sur le concept de passibility hérité de la théologie de Saint Augustin qui désigne une forme de sensibilité d'action différenciant l'homme $\mathrm{du}$ divin. La nature du pouvoir à la fin de la République met aussi en évidence l'intense compétition aristocratique qui aurait détruit le tissu social de la cité. Selon Shakespeare, des personnages comme César et Brutus auraient exalté un mauvais idéal, celui d'une impossible autonomie humaine par rapport au divin. En outre, le sens politique de Julius Caesar insiste sur le début de la dégénérescence de Rome, résultant d'un système de valeurs imparfait.

La deuxième partie concerne Antony and Cleopatra. Le premier chapitre se focalise notamment sur le concept augustinien de libido dominandi et sur la honte, un autre aspect de la vulnérabilité humaine ("The high roman fashion": suicide and stoicism in Antony and Cleopatra, p. I772I9). Diverses attitudes sont analysées, comme celles de Cléopâtre et Brutus. Pour la reine d'Égypte, le suicide serait une 
pratique culturelle spécifique aux yeux des Romains qu'elle qualifie de high roman fashion. C'est une façon de transformer une défaite en victoire, tout en évitant la honte et le jugement moral. Le but recherché est de dégager la cohérence du suicide de Cléopâtre avec sa propre nature. Le dernier chapitre ("A spacious mirror": interpellation and the other in Antony and Cleopatra, p. 220-258) pose en préambule le problème central du jugement moral dans les tragédies. C'est évidemment le cas pour les protagonistes, mais aussi pour le public qui en fait l'expérience. La difficile question de la perception de soi est posée pour des aristocrates comme César et Brutus, ainsi que celle de la relation entre soi et les autres. La conclusion de la deuxième partie (The last interpellation) expose la symbolique chrétienne perçue par le public de la Renaissance. Par exemple, la vie après la mort est mise en avant pour Antoine et Cléopâtre qui pourraient à nouveau se rencontrer. La mort est alors perçue comme un sommeil temporaire. L'ignorance païenne de la révélation chrétienne semble oubliée lorsque Cléopâtre s'interroge sur la conception du suicide source de péché.

La conclusion générale (Between humanism and antihumanism) renoue avec la préoccupation première de l'individualité, véritable fil conducteur de l'ensemble de l'ouvrage. Ensuite, le lecteur trouvera une bibliographie (p. 279-3oo) et un index (p. 3oI-3o8).

Pour conclure, P. Gray offre une réflexion dense et érudite sur la vision shakespearienne de la fin de la République romaine et la romanitas à la Renaissance, tout en maniant culture classique, historique, philosophique et théologique.

Cyrielle Landrea

Université Bretagne Sud cyrielle.landrea@univ-ubs.fr
Mélanie Lozat et Sara Petrella (dir.), La Plume et le calumet. Joseph-François Lafitau et les « sauvages ameriquains », Paris, Classiques Garnier, 20I9, 293 p., $22 € /$ ISBN 9782406087984 .

L'ouvrage, richement illustré, est issu d'un colloque interdisciplinaire tenu à Genève en juin 2016 qui s'est accompagné d'expositions ethnographiques et artistiques. Les onze contributions sont réparties en trois parties (sur le contexte d'écriture, sur son “ Système de religion » et sur les illustrations) auxquelles s'ajoutent deux annexes, une bibliographie, des résumés et index.

En introduction, M. Lozat et S. Petrella rappellent que Lafitau est considéré depuis plus d'un siècle comme un précurseur de l'ethno-anthropologie et, plus récemment, de l'histoire comparée des religions. Elles montrent aussi l'ambiguïté de son travail : les Autochtones, dont la voix est en réalité quasi absente, ne servent qu'à valider la thèse de l'universalisme chrétien. Son utilisation des illustrations rejoint certes l'antiquarisme, mais contribua aussi à façonner un imaginaire du Nouveau Monde qui confortait le sentiment de supériorité des Européens.

P. Borgeaud retrace tout d'abord le parcours littéraire de Lafitau, signalant la naissance de sa méthode comparative dans le Mémoire sur le Ginseng publié six ans avant les Mours. A. Motsch revient ensuite sur l'opinion commune faisant de Lafitau un homme du $\mathrm{XvII}^{\mathrm{e}}$ siècle mais rattachant ses livres aux Lumières. Toute son œuvre tend à prouver l'origine commune des peuples et l'existence d'une unique religion révélée par Dieu. Pour cela il utilise la méthode comparatiste dont l'originalité réside dans le fait que “ les temps anciens » sont le résultat de cette comparaison, ce qui conduit à ensauvager les Anciens et à civiliser les habitants du Nouveau Monde. A. Motsch renverse donc la réception habituelle de Lafitau, mettant au centre la 
dimension religieuse qui avait fait débat lors de sa publication, et soulignant que «Lafitau instrumentalise l'ethnographie justement à des fins apologétiques. » (p. 6I). J.-P. Rubiès poursuit cette entreprise de déconstruction en replaçant les Moeurs dans la tradition apologétique de l'histoire universelle. La comparaison avec Cérémonies et coutumes de J.-F. Bernard publié la même année dans une perspective opposée montre combien Lafitau était « un réactionnaire qui cherchait à rassembler les preuves du consensus gentium à l'aide de l'érudition antiquaire»(p. 71). Pour «réévaluer la nature apologétique des Moeurs » (p. 84), M. A. Kolakowski examine le traitement de la théorie des Moïses et plus généralement le rapport de Lafitau à la Demonstratio evangelica de P.-D. Huet, ce qui le conduit à le rapprocher du figurisme. Cette proximité expliquerait « la réception mitigée » (p. 93) des Mours dans la France des Lumières et sa disparition au xIx $^{\mathrm{e}}$ siècle.

M. Lozat étudie la place dans les Moeurs de la Géographie de Strabon, dont elle rappelle d'abord la redécouverte puis le succès depuis la Renaissance. En effet, Lafitau puise dans la digression sur l'origine des Courètes chez Strabon, qui défend l'unicité de la fonction rituelle chez les différents ministres des dieux, une inspiration pour sa méthode comparatiste et pour son « système de religion ». Dans ce but, "Lafitau accorde une place centrale à la "Pyrolâtrie" ou "Culte du Feu sacré”, dénominateur commun, à l'en croire, de toutes les religions » (p. II4) selon F. Lestringant. Lafitau rapproche ainsi Vestales romaines, cultes solaires américains, mais aussi sacrifices et rituels cannibales afin de défendre sa thèse d'une Révélation universelle. L'analyse synchronique et même anachronique que fait Lafitau de l'Égypte pharaonique et en particulier du hiéroglyphe, sa “ théologie symbolique ", s'inscrit dans ce même système de religion ainsi que le montrent S. D. Brämer et A. Paschoud. L'étude de la représentation figurée des femmes sauvages est l'occasion pour S. Petrella de revenir sur la prétendue ingénuité de la littérature de voyage. Parmi les nombreuses figures de femmes, toujours stéréotypées, on distingue les planches comparatives, dans lesquelles les femmes sauvages évoquent les statues grecques, et les scènes narratives où les figures sont héritées de la tradition médiévale chrétienne censurée par Lafitau afin de réfuter la bestialité des Autochtones. En «neutralisant» les corps, Lafitau met en avant les objets qui seuls caractérisent les Autochtones et les distinguent des Anciens. Pour soutenir ses théories, Lafitau a recours à l'image qui « met l'analogie sous les yeux du lecteur, faisant implicitement passer la ressemblance pour une parenté, voire une identité.»(p. I54), quitte à la manipuler parfois. Ce sont ces arrangements qu'étudie M. Bernhardt à travers les fréquents emplois des gravures tirées des œuvres de Théodore de Bry et de Jean de Léry. Puis P. von Wyss-Giacosa se penche sur les trois planches présentant des “idoles» asiatiques afin d'éclairer le “principe des images synoptiques » à l'œuvre chez Lafitau: en analysant les planches, les lecteurs «seraient conduits à percevoir, de manière sensible, les stades et les développements de l'idolâtrie [...] et à les lire, aidés par les légendes de Lafitau, à un niveau symbolique permettant invariablement de remonter aux vestiges de la vraie religion. »(p. I77). Enfin, M. Marrache-Gouraud réfute l'idée répandue à la suite de $\mathrm{M}$. de Certeau selon laquelle dans les Mours Lafitau exposait un catalogue de curiosités tout en reconnaissant qu'il entendait "se faire passer pour un “curieux" » (p. I85).

Loin d'être hagiographique, cette relecture desMours de Lafitau, le réinsérant dans son contexte, lui redonnant une épaisseur et replaçant l'histoire religieuse au cœur de son travail, est stimulante. Cette plongée dans les controverses qui précèdent les Lumières nous offre une réflexion épistémologique salutaire. Un 
bel ouvrage en somme, très clair, qui mérite d'être lu en attendant l'édition critique française des Moeurs que préparent A. Motsch, G. Tissot et R. Melançon.

\section{Clément Bur Institut National Universitaire Champollion (Albi) clement.bur@univ-jfc.fr}

Bartolo Natoli et Steven Hunt (éds.), Teaching Classics with Technology, Londres et New York, Bloomsbury Academic, 20I9, 264p., $£ 75.00$ / ISBN 978 35 oirog39 (hardback),

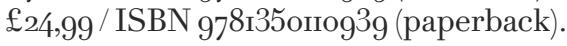

“Enseigner les Classics avec la technologie ", c'est avant tout changer et adapter ses pratiques, ses outils et ses méthodes d'éducation à l'ère - en constante évolution - du numérique; il ne s'agit pas d'y voir un obstacle mais une réelle opportunité. En d'autres termes, c'est partager, mettre en réseau, échanger et débattre pour améliorer, encore et toujours, la qualité de l'enseignement des civilisations et langues anciennes tout en rendant leur apprentissage plus accessible et attractif pour les élèves et étudiants. L'objectif principal de l'ouvrage de Bartolo Natoli et Steven Hunt est de donner les clefs essentielles aux enseignants de tous niveaux pour suivre l'évolution extrêmement rapide du domaine digital, bien plus accessible et compris des étudiants hyper connectés dont il faut, à cet égard, acquérir le même niveau de compétences. “S'adapter ou mourir » (p. xi), tel est effectivement l'enjeu vital qui pèse, de plus en plus lourdement, sur les Classicists.

Ainsi les dix-neuf contributions s'enchaînent dans un processus progressif, similaire à celui de l'apprentissage: la première partie se concentre sur la théorie tandis que les deux suivantes exposent des exemples pratiques et des études de cas. Ce sont donc d'abord les nouveaux modèles digitaux d'apprentissage des Classics qui sont expliqués, ainsi que leur intérêt pour ce domaine d'étude. Les contributions donnent en effet une vision panoramique des ressources en ligne disponibles, comme le fait Alan Chadwick qui explique les avantages d'utiliser des sites internet de cours en ligne, comme CyberCaesar pour le latin. Stephen Slota et Kevin Ballestrini introduisent quant à eux les nouveaux supports numériques de l'enseignement tels les jeux, notamment celui issu de l'Operation LIAPIS, quand Elizabeth Lewis montre tous les bienfaits d'adapter à l'univers des Classics des applications déjà existantes comme Microsoft OneDrive. Tous ces modèles théoriques, mais pas seulement, sont ensuite appliqués de manière très réussie en salle de classe dans laquelle le lecteur est plongé. Les contributeurs insistent d'abord sur l'application des techniques numériques à l'enseignement de l'art et de l'histoire des mondes antiques. Ray Laurence, par exemple, indique comment YouTube devient un outil puissant de sensibilisation à ces domaines d'études. D'autres contributions révèlent comment certains outils numériques, grâce à leur caractère surtout visuel, transforment les cours et engagent davantage les élèves: Sonya Nevin se sert de vases grecs animés virtuellement, Matthew Nicholls de modélisations 3D de bâtiments d'architecture romaine, Caron Downes d'IPad pour visualiser le théâtre grec. Enfin, dans la dernière partie du livre, c'est plus précisément l'usage de la technologie dans l'apprentissage des langues anciennes qui est mis en lumière. Plus particulièrement, Steven Hunt signale comment le tableau blanc numérique accentue le dialogue interactif et social lors du cours à l'université, mais aussi comment certains services en ligne, tel Google Docs exposé par Roger Travis, se meuvent en créateurs 
de communauté pour les Classicists, alors tous réunis virtuellement pour commenter collaborativement un document ancien. La communication de Lisa Hay, qui clôt cet ouvrage, montre la flexibilité et la diversité des enseignements et des apprentissages autorisés par la technologie, ainsi que leur capacité d'adaptation aux classes de Classics. Les dernières parties révèlent donc, à tous les niveaux d'études, tout l'engouement suscité par l'utilisation de ces nouvelles techniques, rejaillissant sur les Classics eux-mêmes.

Le livre de Bartolo Natoli et de Steven Hunt s'inscrit dans une problématique et une historiographie aussi récentes que le e-learning, effectivement initiées vers la fin des années r99o. Dans la continuité des travaux de leurs prédécesseurs, notamment de l'ouvrage primordial de Richard Lafleur (Latin for the 2Ist Century: From Concept to Classroom, I998), les auteurs s'intéressent aux nouvelles méthodes pédagogiques appliquées à l'enseignement des langues anciennes. Mais leur ouvrage est aussi innovant: il ne s'est pas restreint à l'usage des outils technologiques dans les classes de langues anciennes mais intègre aussi toute une réflexion sur les ressources digitales utilisées dans les enseignements de géographie, d'histoire et d'histoire de l'art des mondes antiques jusque-là largement laissés de côté. Les auteurs ont aussi élargi le champ d'études, ne le limitant plus aux seuls États-Unis. Cependant, malgré la volonté affichée par les éditeurs du volume d'offrir un panorama mondial de l'utilisation des techniques pédagogiques numériques, on peut regretter que le champ de réflexion soit en fait restreint au monde anglophone (États-Unis, Royaume-Uni, Australie). En outre, on note que l'enseignement du grec est largement laissé de côté, ce qui est assumé par les auteurs, alors que les ressources digitales pour cette langue sont aussi très nombreuses : entre autres, les bases de données iconographiques comme les Beazley Archives ou encore, pour parler aussi de l'enseignement supérieur, les bases de données textuelles telles que le TLG (Thesaurus Linguae Graecae), qui recensent les textes antiques et permettent de nombreuses recherches et analyses lexicales.

Nous pouvons enfin regretter l'absence, dans ce livre, de multiples outils de vulgarisation scientifique des Classics, qui suscitent tout autant d'enthousiasme chez les élèves, même davantage : certains blogues, comme classicalstudies.org, tenus par des chercheurs spécialisés ou des laboratoires de recherche, peuvent constituer une approche captivante, en classe, pour des élèves de tous niveaux. Surtout, l'ouvrage semble négliger l'importance, pour les collégiens, lycéens ou étudiants, de l'attractivité des réseaux sociaux. Pour les Classics, nous pouvons nous attarder sur l'importance de Twitter, outil très apprécié du monde de l'enseignement et de la recherche: on remarque d'ailleurs que les éditeurs du volume ont jugé utile de mentionner, pour chaque contributeur, son nom d'utilisateur (toujours précédé d'un @) sur ce réseau social, mais sans en expliquer vraiment la raison. Pour prendre un exemple français, le compte @Antiquipop dédié à la réception de l'Antiquité dans la culture pop contemporaine peut constituer un support de cours attractif pour des élèves de tous niveaux: des chercheurs y analysent la publicité Ferrero Rocher qui met en scène les dieux de l'Olympe, ou encore un clip de Katy Perry, Dark Horse, dans lequel la chanteuse apparaît en Cléopâtre. Pour le monde anglo-saxon, des comptes comme @RockingClassics, dédié au détournement de paroles de musiques récentes alors transcrites en latin, mais aussi le compte de la maison d'édition de cet ouvrage, @ BloomsburyClass, qui raconte des mythes grecs entièrement par emojis, participent de l'engouement nouveau suscité par les Classics grâce à la technologie. Plus encore, le compte@GreekHistoryPod mêle deux 
nouveaux médias susceptibles d'intéresser tous les élèves: d'abord le réseau social puisqu'il propose des tweets quotidiens sur les ressources des Classics, mais aussi les podcasts, c'est-à-dire des enregistrements audios (souvent sous forme d'interviews) sur des sujets particuliers, qui peuvent tout à fait constituer des supports de cours pour les enseignants et/ou être écoutés par les élèves pour compléter le cours de leurs professeurs.

Ces quelques exemples montrent bien qu'un livre exhaustif, dressant la liste complète des ressources numériques disponibles (et en mouvement constant) pour les Classics, n'est pas imaginable et pour le coup, pourrait faire l'objet d'un site internet. Néanmoins, l'ouvrage collectif édité par Bartolo Natoli et Steven Hunt permet, à maints égards, de mieux comprendre la nécessité d'adaptation de l'enseignement des Classics à l'ère du numérique et du collectif, et prodigue aux professeurs de précieux conseils pédagogiques, théoriques et pratiques, pour susciter un regain d'intérêt pour les mondes anciens chez les élèves.

Léa Marques

Université de Paris lm.leamarques@gmail.com

\section{S. Douglas Otson (éd.), Ancient Comedy and Reception. Essays in Honor of Jeffrey Henderson, Berlin, De Gruyter, 20I3, Io86 p., £240 / ISBN 978I6I/45ı663.}

Le volume Ancient Comedy and Reception est l'énorme fruit - 1086 pages - d'une aventure complexe qui explique en partie ses limites. Consacré à la comédie ancienne et à sa réception, comme l'indique le titre, il embrasse un domaine très large et loin d'être homogène: celui de la comédie ancienne, au sens large de comédie composée dans l'Antiquité : la comédie grecque (ancienne au sens strict, moyenne et nouvelle), latine (Plaute et Térence), mais aussi d'autres auteurs dont les textes ont été largement perdus, et leurs réceptions. Traiter de tous ces auteurs ensemble ne va pas forcément de soi; traiter en plus de leurs réceptions, très différentes, est une démarche intéressante du point de vue de l'histoire du genre comique.

L'ouvrage n'est hélas pas doté d'une introduction qui permettrait de tisser des liens entre les cinquante contributions ici réunies : à charge pour le lecteur, donc, de les mettre en relation, ou de n'aller chercher que ce qui l'intéresse - et il aura de quoi faire, car plusieurs contributions sont tout à fait passionnantes et de haut niveau. Si elle est dépourvue d'introduction - ainsi que de conclusion - cette collection de contributions est cependant précédée d'une courte préface d'une demi page (Foreword) signée de l'éditeur du volume, $\mathrm{S}$. Douglas Olson, qui éclaire quelque peu cet aspect composite : l'ouvrage a été conçu par Wolfgang Haase qui pour des raisons de santé n'a pas pu le mener à son terme, et S. Douglas Olson n'a fait qu'éditer ce dont il a hérité, à savoir une série de contributions. Si bien que ni le concept d'Ancient comedy, ni celui de reception ne sont interrogés. Très rares sont enfin les liens explicites avec l'œuvre de Jeffrey Henderson, à qui est dédié ce volume d'hommages.

Le volume est composé de trois parties. La première, intitulée Ancient Comedy and Receptions, regroupe des travaux sur les comiques et leur réception ancienne: neuf portent sur Aristophane, quatre sur Ménandre, deux sur Plaute, à quoi s'ajoutent des travaux plus transversaux - notamment sur la comédie ancienne au sens strict à Rome, dans les romans, ou encore chez Lucien. La contribution de Simone Beta pose la question de l'influence d'Aristophane sur la comédie à Rome à travers la figure de Gnaeus Naevius, dont il nous reste quelques fragments, titres et résumés d'intrigues. Il démontre 
que le poète fait figure d'exception, lui qui, selon Aulu-Gelle, fut emprisonné pour ses maledicentia et probra - des traits caractéristiques d'Aristophane. Même s'il ne nommait pas ses cibles à la manière du poète grec, elles étaient tout à fait reconnaissables. Les intrigues aussi semblent témoigner d'un lien assumé avec la comédie ancienne, et notamment les Nuées. Enfin Beta pointe des correspondances lexicales tout à fait convaincantes. La contribution d'Ian Ruffell porte également sur le monde romain et dresse un long et utile panorama de la réception de la comédie ancienne dans les textes latins, en réévaluant notamment le rôle de la réception rhétorique. Les références à la comédie ancienne chez Cicéron, Quintilien, Horace sont d'abord passées en revues : si on peut lire chez Horace l'influence des travaux hellénistiques, qui soulignent l'excessive liberté et l'agressivité de la comédie ancienne, lui préférant Ménandre, Cicéron et Quintilien en donnent une vision plus positive. La comédie ancienne représente pour eux un modèle stylistique et moral, alliant un style agréable (suavis) à un propos sérieux (gravis). Et Aristophane est qualifié de facetissimus poeta veteris comoediae par Cicéron, une formulation qui sera reprise à la Renaissance. Si l'agressivité et le goût pour l'invective de la comédie ancienne ne sont pas ignorées, elles apparaissent chez ces auteurs comme mises au service d'une attitude morale : il s'agit de s'attaquer aux vices des contemporains, ce qui la rapproche de l'art oratoire. Ruffell évoque ensuite le recours des auteurs latins à la comédie ancienne comme répertoire d'exemples historiques et notamment politiques, un des plus fréquents, avec Socrate, étant le Périclès comique tonnant tel Zeus. La comédie ancienne est également convoquée dans l'élaboration de la satire. Mais de manière générale Ruffell souligne que les textes de la comédie ancienne sont très peu connus, et que, mis à part Aulu-Gelle, les auteurs latins se limitent à des exemples récurrents. Surtout, cette connaissance se limite aux textes. Enfin, Ruffell revient sur la quasi absence d'influence de la comédie ancienne sur la comédie à Rome.

La deuxième partie porte sur les réceptions de la comédie au Moyen Âge, à la Renaissance et durant la Première modernité. Cette fois c'est la comédie latine qui est le mieux illustrée, puisque seules quatre contributions sur douze portent sur la comédie grecque. Ludovica Radif présente rapidement quelques approches d'Aristophane dans l'Italie du $\mathrm{xv}^{\mathrm{e}}$ siècle omettant celles d'Alessandro d'Otranto et de Franciscus Passius - et rapporte ses tentatives de mettre en scène l'adaptation de la Fabula Penia de Rinucci et de la comédie perdue de Machiavel Le Maschere, une démarche de recherche-création tout à fait intéressante. John Nassichuk compare deux traductions latines des Nuées, celle de Divo et celle de Frischlin, en se concentrant sur certains néologismes se trouvant dans les propos de Strepsiade, faisant l'hypothèse que la traduction de Divo sert à identifier le lexique tandis que celle de Frischlin est plus attentive au sens, ce qui ne saurait être contesté - je renvoie ici aux analyses de Simone Beta sur la traduction de Divo (Anabases 2I, 2015 et QUCC 2012). La très riche contribution de Robert Miola porte sur la réception d'Aristophane en Angleterre de I5oo à I66o ; elle passe en revue l'importance d'un Aristophane pédagogique à usage des apprentis hellénistes; la cristallisation de l'interprétation de son œuvre, et notamment des Nuées, autour de la question de la satire personnelle, et deux traductions en anglais du Ploutos du xviı ${ }^{\mathrm{e}}$, avant de revenir sur la présence d'Aristophane chez Ben Jonson. Le début de la contribution, de seconde main comme le signale très honnêtement l'auteur, comporte un certain nombre d'approximations: ainsi, aucune source n'atteste une mise en scène du Ploutos par les membres de la Pléiade en i56o. Parmi les auteurs anciens qui ont retravaillé l'œuvre 
d'Aristophane, c'est Horace, qui voit en Aristophane avant tout un satiriste, qui est le plus lu dans l'Angleterre de la Première modernité. Particulièrement intéressantes sont les analyses qui montrent comment les traductions du Ploutos tentent de rendre la comédie compatible avec la morale chrétienne. L'article de Benjamin Garcia Hernandez, Rosario Lopez Gregoris et Carmen Gonzalez Vazquez propose une belle synthèse collective de la réception de Plaute et Térence dans la littérature espagnole.

À côté de ces synthèses, d'autres contributions portent sur un auteur, voire sur une œuvre en particulier. On noteral'importance de Plaute pour la période : Laura Kendrick s'intéresse au devenir de l'Amphitryon de Plaute au Moyen Âge, Hélène CasanovaRobin à l'influence de Plaute sur Pontano ; Térence est cependant présent également, avec Maik Goth dont la contribution porte sur le devenir de l'Andrienne de Térence chez Stelle et Bellamy; Philippe Ford et Adele Scafuro s'intéressent à celle des deux comiques latins sur Molière, et Adele Scafuro sur Titus Andronicus. On regrettera que les comiques latins n'aient pas donné lieu à une large synthèse à la manière de celle d'Hernandez, Lopez Gregoris et Gonzalez Vazquez pour les littératures de langue anglaise, italienne et française. De la même manière, la réception d'Aristophane en dehors de l'Angleterre est dans cette partie très largement ignorée.

La troisième et dernière partie fait en revanche la part belle à Aristophane, avec treize contributions sur dix-huit; elle varie par ailleurs davantage les aires de réception. Maria Luisa Chirico s'intéresse aux traductions d'Aristophane dans l'Italie du xix ${ }^{e}$ s.; Gonda Van Steen au burlesque mythologique à Athènes à la fin du xix e et à son recours à Aristophane; Kevin Wetmore à la réception africaine-américaine de Lysistrata. On lira avec intérêt les contributions de Marina Kotamani, Lysistrata on Broadway, et Simone Beta sur la réception d'Aristophane dans le modern musical theater. Très originales également sont les contributions d'Amanda Wrigley, Aristophanes at the $B B C$, et de Martin Winkler sur Aristophane au cinéma.

Ainsi, le volume fournit plusieurs contributions de très grande valeur, mais peu, hélas, de synthèses qui feront, espérons-le, l'objet de travaux ultérieurs.

\section{Malika Bastin-Hаммоu Université Grenoble Alpes malika.bastin@univ-grenoble-alpes.fr}

\author{
Mark A. Ralkowski, Plato's Trial \\ of Athens, Londres et New York, \\ Bloomsbury Academic, 20I9, 248 p., \\ $£_{90} /$ ISBN 978I474227247 (hardback),

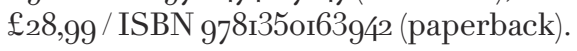

Quelle est la relation que Socrate entretenait avec la démocratie athénienne? Peut-on comprendre les raisons de son procès et sa condamnation à mort en étudiant la place qui a été la sienne au sein de la cité ? Son accusation a-t-elle été avant tout politique ou religieuse?

Pour répondre à ces questions, Mark Ralkowski utilise différents textes de Platon, depuis l'Apologie de Socrate au Banquet en passant par la République ou le Gorgias, et montre de quelle façon les figures de Socrate permettent de faire le procès de la démocratie athénienne. Si l'ouvrage semble démarrer avec le ton du roman policier, notamment en interrogeant le mobile de la condamnation du philosophe, le suspense est très rapidement levé. Dès le départ, on sait que Socrate a payé de sa vie la volonté d'amener l'âme des citoyens athéniens vers la sagesse, notamment en attaquant les sophistes et en condamnant les excès d'une cité soumises aux tentations impérialistes.

Tout en prenant au sérieux les réflexions nietzschéennes sur Socrate, Mark Ralkowski montre néanmoins que ce dernier 
ne voulait pas mourir. Il a été condamné par un régime qui n'appréciait guère son mode de vie philosophique et son refus de rallier la pensée dominante. Cette condamnation ne signifie pas une quelconque attirance de Socrate pour l'oligarchie ou bien une attitude impie dont il se serait rendu coupable, et dont la démocratie athénienne de son époque ne se souciait guère. À partir d'une fine restitution de la littérature scientifique existante, le premier chapitre remet en cause la thèse de la condamnation pour impiété sans pour autant donner raison à ceux qui plaident en faveur de la dimension politique de la condamnation.

Mark Ralkowski pense qu'il faut aller voir dans d'autres textes que l'Apologie pour saisir de quoi est-ce que l'on accusait Socrate. La réponse de l'énigme est non pas dans un mais plusieurs textes. L'Apologie de Socrate rend compte du procès et des paroles de Socrate mais d'autres textes de Platon éclairent d'une lumière nouvelle ce qui se jouait à ce moment-là entre les différents acteurs de la cité. Dans $L e$ banquet, les dialogues entre Alcibiade et Socrate permettent de saisir l'un des reproches adressés au philosophe. En confrontant les désirs d'Alcibiade, dont la vie fut constituée d'excès en tout genre, et la modération de Socrate quant aux plaisirs du corps, Platon souligne l'opposition de deux modes de vie antagonistes. Celui $\mathrm{du}$ philosophe ne peut être compatible avec celui du jouisseur, omniprésent dans une démocratie athénienne minée de l'intérieur par ses ambitions de conquête. À un Alcibiade lui faisant explicitement des avances, en soulignant toutes ses qualités intellectuelles, Socrate fait comprendre qu'il ne peut pas désirer en même temps le philosophe et la philosophie. Socrate lui dit qu'il doit choisir entre deux modes de vie distincts (celui du philosophe ou celui du jouisseur), quand bien même ce choix est tragique; et s'il choisit le mode de vie philosophique, il doit mener une vie bonne et modérée, cherchant à s'affranchir de la tyrannie des sens pour aller vers le monde de l'intellect. Le discours de Diotime à la fin du Banquet au sujet de la voie à suivre pour aller de l'attirance pour les beaux corps jusqu'à l'attirance pour les belles connaissances, notamment celles relatives à l'idée du beau, prend tout son sens en relation avec les deux modes de vie antagonistes décrit au cours des échanges entre Socrate et Alcibiade.

Ce point réapparait dans le Gorgias où la “ vie philosophique » de Socrate est opposée à la "vie politique » du sophiste Calliclès. Le premier poursuit la vérité et la sagesse alors que le second cherche les honneurs et le pouvoir. Selon Mark Ralkowski, Socrate ne peut être accusé de corrompre la jeunesse. Toute sa vie, il a tenté de convertir celle-ci au mode de vie philosophique et de s'opposer au relativisme moral et au scepticisme régnant sous l'ère de Périclès (p. 92). Pour Platon, le véritable corrupteur de la jeunesse n'est pas Socrate mais la démocratie athénienne. Et c'est cette "thérapeutie philosophique» qui a été condamnée lors du procès. L'Apologie, Le banquet et Gorgias ont posé le diagnostic du mal et La République a proposé le remède, notamment en évoquant le gouvernement de la cité par les philosophes capables de percevoir ce qu'est la justice grâce à leur intellect. En lisant certains passages du livre de Mark Ralkowski, on ne peut s'empêcher de penser aux pages lumineuses de Henri Bergson consacrées à Socrate dans Les deux sources de la morale et de la religion (Paris, PUF, ı984, p. 59-63 [ı932]). Si le philosophe athénien semble accorder une place importante à la raison, Bergson pense que son enseignement relève davantage de la posture mystique. Cette vie philosophique serait-elle à la frontière entre rationalisme exigeant et spiritualité? Si c'était le cas, il ne s'agirait dès lors non plus d'opposer les modes de vie de Socrate et de Calliclès mais de regarder aussi ce qui peut les rapprocher, notamment du point de vue du désir et du souci de soi. 
L'une des qualités de l'ouvrage de Mark Ralkowski est au niveau de l'intertextualité sans cesse mobilisée. Celle-ci a le mérite à la fois de contextualiser le procès de Socrate tout en conceptualisant autour du mode de vie philosophique défendu par Platon face à la corruption de la cité. Si l'on réduit bien souvent la pensée de Platon à son essentialisme ou à sa dimension pseudo-totalitaire soulignée de manière caricaturale par Karl Popper dans La société ouverte et ses ennemis (1945), le livre de Mark Ralkowski montre l'apport heuristique du philosophe grec à la fois au niveau de son aspiration à atteindre la sagesse et dans son refus du relativisme des points de vue exprimés par les sophistes. Dans le contexte actuel de la pandémie Covid ıg, où n'importe qui peut donner publiquement son avis sur la Chloroquine, prévoir à partir de modélisations mathématiques la fin de la pandémie ou bien proclamer la nécessité ou pas du confinement, les sirènes d'alarme lancées par Platon sur les apories de la rhétorique - à ne pas confondre avec la rigueur de l'argumentation intellectuelle - sont d'une grande actualité. Ses propos à l'égard de la rivalité entre les prétendants à la vérité sont également d'une grande utilité dans un monde où la multiplication de discours scientifiques sur le Covid i9 est omniprésente au sein de ce que l'on pourrait appeler avec Pierre Bourdieu «le champ scientifique ». Sans en faire non plus la solution miracle, la figure du philosopheroi, capable de gouverner la cité non pas en fonction de ses désirs ou de son courage mais à partir de son intellect, n'est peutêtre pas si obsolète qu'elle en a l'air à un moment où il est nécessaire de s'interroger sur la place de la justice dans la cité et sur les mesures visant au bien commun des citoyens.

Jean ZAGANIARIS

Lycée Descartes de Rabat zaganiaris@yahoo.fr
Brett M. Rogers et Benjamin Eldon Stevens (ed.), Once and Future Antiquities in Science Fiction and Fantasy, Londres et New York, Bloomsbury Academic, 20I9, 248 p., £65 / ISBN 978I35oo68940 (hardback), £ı9,99 / ISBN 978I35007488o (paperback).

L'ouvrage est le fruit des travaux entamés lors d'une conférence à l'University of Puget Sound, Tacoma, en mars 20I5. Son point de départ est la conviction que l'on retrouve une forme épistémologique similaire de displacement (ou estrangement) dans la Science-Fiction et Fantasy (SF\&F) et dans les études classiques: reconstructing the past is similar to imagining alternative worlds, requiring interpretive paradigms that differ from lived experience and from contemporary truth-claims about the "real" (p. 9I).

T. Keen commence par une réflexion théorique en revenant sur son article pionnier de 2006 «Models and Methodologies of Classical Reception in Science Fiction». Il inventoriait sept degrés d'emprunt (appropriation, interaction, borrowing, stealing, retellings, allusion et ghosting) auxquels s'ajoutent désormais les cinq catégories de C. W. Marshall (cosmetic reference, indirect reference, envisioning, revisioning et engagement) se rapportant aux attentes de l'audience et à sa capacité à apprécier la réception. Puis J. Weiner s'appuiesurGradiva deW. Jensen(Igo3) pour souligner la proximité entre l'archéologie et la fantasy puisqu'en (re)créant un monde, l'une et l'autre reflètent leur époque tout en opérant un displacement. Pour L. Zientek, dans The Engine of Gods (1994), dans lequel des exo-archéologues partent à la recherche d'une civilisation extraterrestre perdue (dont la langue est appelée linéaire C) à bord du vaisseau Winckelmann, J. McDevitt invigorates, then re-examines, the desire to re-enact the past that was so prevalent in the early practice of classical archaeology, especially for Schliemann 
(p. 57). V. Tomasso montre, lui, comment la présence des dieux dans Soldier of the Mist de G. Wolfe (I986) brouille les frontières entre fiction historique et fantasy. De la sorte il entend to flesh out his conception of the ancient Greek world (p. I78), dans lequel les dieux apparaissaient parfois aux mortels. Ces jeux d'emprunts livrent une réflexion épistémologique originale sur les études classiques.

L'Odyssée est à l'honneur puisque $\mathrm{O}$. Knorr et $\mathrm{S}$. Lye considèrent respectivement que His Dark Materials (1995-200o) de P. Pullman et Le Voyage de Chihiro de H. Miyazaki (20or) en sont des adaptations tandis que F. Foster examine les emprunts aux rituels nécromantiques de l'Odyssée dans Time of the Ghost de D. W. Jones (I98I). C. Kenward explore la réception d'Homère dans la culture populaire et la rationalisation des mythes dans la $\mathrm{SF} \& \mathrm{~F}$ à travers la série Dr. Who Myth Makers (I965) et le comics Thor Annual (I979) qui mêlent voyages temporels et guerre de Troie. B. E. Stevens dévoile enfin les emprunts aux mythes classiques (Icare, Orphée, Eurydice...) dans les œuvres d'H. Oyeyemi.

Dans la seule (et très intéressante) analyse liant l'œuvre au contexte social, S. B. Moses et B. M. Rogers interprètent la figure d'Atlas dans le Rocky Horror Picture Show (1975) comme une manière to respond to and critique modern norms of hegemonic masculinity embodied and promulgated by Charles Atlas et par les péplums (p. Iog), et donc comme une défense de la culture queer. J. C. Rank compare, elle, la figure de Cassandre, considered by Karin Beeler as a "mythic prototype" for "women of vision" in postmodern science-fiction (p. I35), dans l'Agamemnon d'Eschyle avec la policière Kiera, l'héroïne de la série télévisée Continuum (2012-2015). Bien que cette proximité ne semble pas voulue (elle est l'une des rares à poser la question, p. 136-I37), le rapprochement est éclairant, notamment sur les figures féminines de la SF\&F.
C. W. Marshall étudie la présentation (et naturalisation!) des monstres antiques dans les différentes éditions du bestiaire de Donjons \& Dragons, et y observe une réception indirecte façonnée par les contraintes ludiques. Après avoir montré combien l'univers de Warhammer $40 K$ s'inspire de l'Empire romain, notamment la figure de l'empereur dans la série de romans Horus Heresy qui rappelle celle d'Auguste dans l'Énéide, A. McAuley se penche sur la biographie des premiers auteurs de $40 K$, familiers des études classiques, et sur le processus collaboratif d'écriture afin de mieux comprendre une réception complexe et consciente.

T. Keen concluait le premier article en indiquant cinq défis àrelever :l'impossibilité d'embrasser toute la SF\&F, le danger de surinterpréter, le manque de théorisation et de prise en compte de la littérature SF \&F et scientifique non anglophone, la nécessaire formation à la critique SF\&F. Qu'en est-il pour ce volume? Le principal défaut me semble être le manque quantitatif. Nous avons une juxtaposition d'études de cas (pas toujours d'œuvres les plus connues), dans lesquelles l'accent est généralement mis sur une analyse de la structure du récit et un commentaire artistique impliquant un résumé substantiel et parfois une surinterprétation. De telles études ne sont guère profitables à ceux qui ne connaissent pas l'œuvre choisie, notamment parce qu'elles ne permettent pas une montée en généralité. La réflexion sur le displacement est parfois un peu artificielle, tout comme le plan du livre, et on regrette que les auteurs n'aient pas tiré davantage profit des catégories de T.Keen ou de C.W. Marshall exposées dans le chapitre I. Ainsi la formation et le parcours de l'auteur expliquant de tels emprunts, leur réception par le public, le contexte de l'œuvre pouvant expliquer tel ou tel choix, toutes ces questions sont trop souvent ignorées. Enfin, les travaux se limitent presque exclusivement au monde anglo-saxon, 
ce que regrettait déjà T. Keen : I know of no other field of classical studies in which writing in German, French, and Italian is so completely ignored (p. I4). Cet ouvrage confirme néanmoins la richesse de la SF\&F pour les études réceptionnistes.

Clément Bur Institut National Universitaire Champollion (Albi) clement.bur@univ-jfc.fr

Stine Schierup (éd.), Documenting Ancient Rhodes: Archaeological Expeditions and Rhodian Antiquities, Aarhus, Aarhus University Press, 20I9, 332 p., 349,95 kr / ISBN 9788771249873.

La maggiore isola del Dodecaneso fu notoriamente un centro politico, economico e culturale di primaria importanza nel corso dell'intero evo antico. Questa importanza rimase ben presente anche nei secoli posteriori all'Antichità; soprattutto nei secoli della dominazione ottomana (I522-I9I2), Rodi assunse anche la funzione e il carattere di un importante lieu de mémoire - tanto della civiltà greca, sussunta nel simbolo della "meraviglia" che esistette sull'isola, il Colosso, quanto della cristianità, rappresentata dagli Ospitalieri che avevano controllato Rodi dal i3og alla conquista ottomana e dai loro monumenti. Proprio questo ruolo di Rodi nella memoria culturale europea rende la storia dei viaggi, delle descrizioni e degli scavi archeologici sull'isola particolarmente interessante - si tratta però di un argomento ad oggi studiato poco ed in modo molto saltuario. È dunque una pregevole iniziativa quella che ha portato, nel 20I7, all'organizzazione presso il Museo Nazionale Danese di un convegno dedicato agli scavi archeologici del xix e del primo xx secolo sull'isola, e di cui il volume oggetto di questa recensione rappresenta gli atti.
Non è sorprendente che l'iniziativa venga dalla Danimarca, visto che gli scavi danesi, finanziati dalla fondazione Carlsberg, che si svolsero tra il Igo2 e il IgI4, rappresentano uno dei momenti cruciali nell'esplorazione dell'isola e che, in conseguenza, numerosi materiali rodii presero la via della Danimarca. Se la storia degli scavi (anche danesi) sull'isola è stata già oggetto di studi di notevole valore negli ultimi anni - anche grazie alla grande mostra su Rodi ospitata al Louvre nel 2014-20I5 e al catalogo di accompagnamento - è lodevole l'intenzione del volume di presentare una serie di studi sugli scavi in una prospettiva "pan-europea", integrata da riflessioni sulla conservazione di materiali rodii in diversi musei (British Museum, Louvre, Museo Archeologico Nazionale di Firenze), sui resoconti di viaggio e di scavo, sui "contatti" con gli scavi clandestini e il mercato antiquario. Particolarmente interessanti sono in questo senso gli spunti relativi alle modalità di circolazione del materiale archeologico, centrali per poter affrontare i problemi, ancora molto numerosi, relativi alla provenienza di oggetti custoditi nei musei europei. In particolare, alcuni riferimenti al fatto che, al di là degli scavi e delle vendite, si debbano considerare in modo molto più sistematico le reti sociali degli archeologi e di chi cooperava con loro, i diversi meccanismi di scambio e per esempio le pratiche di concessioni o scambio di doni, sono da contare tra i contributi più significativi di questo volume, insieme ad alcune importanti pagine di metodologia sui problemi e le strategie per poter studiare le pratiche archeologiche del xix secolo (in particolare negli articoli di A. Villing, "The Archaeology of Rhodes and the British Museum: Facing the Challenges of igth-Century Excavations", 7I-95, e di N. Salmon, "Archives and Attribution: Reconstructing the British Museum's Excavations of Kamiros", 97-II2). Degno di menzione è poi l'articolo di B.B. Rasmussen su Helvig Kinch, pittrice e 
moglie dell'archeologo Karl F. Kinch, che prese parte alla missione archeologica come disegnatrice; il contributo ne presenta in modo eccellente la vita e la carriera, i contatti con l'archeologia e l'importanza nella diffusione di immagini di Rodi, ma anche l'integrazione di questi disegni e dei dipinti rodii nel più ampio contesto della sua attività pittorica.

Purtroppo, però, il volume non riesce a mantenere costantemente ciò che promette. Il problema più grande è di coerenza: l'insieme degli articoli non si concentra, come si immaginerebbe dalla breve introduzione dell'editrice (9-I4) sulla storia degli studi e sulla storia dell'archeologia, o sulla storia del turismo e sul ruolo di Rodi nell'immaginario europeo. Gli studi sull'Italia, ad esempio, si concentrano pochissimo sugli aspetti "politici” degli scavi italiani a Rodi, anche legati alla legittimazione del dominio sull'isola e visibili, tra le altre cose, nell'esaltazione del legame con Rodi di Casa Savoia (come nella paraetimologia del motto della dinastia, FERT, interpretato già nel Cinquecento come fortitudo eius Rhodum tenet - temi già affrontati in letteratura, in particolare da M. Livadiotti e L. Ciacci, che avrebbero però qui meritato maggiore approfondimento.

Molti contributi, invece, si rilevano "tradizionali" contributi archeologici, che presentano gli oggetti contenuti in collezioni di materiali rodii senza investigarne in modo particolare la composizione e la provenienza (la collezione rodia a Lund, per esempio, è introdotta in poche pagine da K. Göransson, che traccia ne traccia la storia, 2I8-220, ma i singoli vasi che la compongono sono presentati in un lungo catalogo da G. Bourogiannis, 222-239, senza che appia chiaro cosa questo abbia a che fare con la storia dell'archeologia di Rodi), o che discutono singoli aspetti delle pratiche culturali, cultuali, religiose, ecc. di Rodi antica. Ancora una volta è esemplare l'articolo di Salmon, che appare composto di due parti completamente scollegate tra loro: quella, già menzionato, sui metodi per studiare scavi del XIX secolo e uno studio di materiali ceramici rinvenuti a Kamiros.

L'immancabile articolo sul Colosso di Rodi (L.W. Sørensen, "The Colossus of Rhodes: A Powerful Enigma", I5-34) non può ovviamente fornire una completa storia delle ricostruzioni del Colosso, o della sua ricezione, temi per cui non basterebbe un libro intero. Non riesce però a fornire una selezione coerente o motivata e procede in modo caotico da disegni medievali a ricostruzioni moderne, all'uso del Colosso in caricature e pubblicità, a progetti moderni, senza che sia chiaro in alcun momento cosa - a parte il Colosso - dovrebbe dare unità al contributo. Meglio riuscito il capitolo su "Early Explorers of Rhodes I342-I853" (N. Badoud, 35-5o), che non riesce però a muoversi oltre un puro livello narrativo, e non sottolinea a sufficienza quello che dovrebbe essere uno dei punti cruciali dell'intero volume: perché Rodi fosse così importante nell'immaginario dei viaggiatori europei, e quale ruolo l'isola giocasse nella memoria culturale "occidentale".

Il volume contiene numerose illustrazioni a colori di grande qualità - una caratteristica rara e da sottolineare con forza. Meno attenta è stata però la cura editoriale: numerosi sono i refusi, e molto spesso l'inglese degli autori non madrelingua non è stato rivisto con sufficiente cura, generando errori marchiani come "comparative" per "comparable" (p. I53) o "cruxifiction" (p. 199). Ma anche più in generale si trovano errori che si potrebbero evitare con cura maggiore, come nel caso dell'espressione "last century" ad indicare il xix e non il xx! A. Di Rosa ("From Rhodes to Rome: The Archaeological Objects Discovered by Gian Giacomo Porro in the Early 2oth Century", I45-I57) colloca Mortara e Bobbio in Sardegna (o nel Regno di Sardegna, benché si tratti del xx secolo, ed è difficile dire cosa sarebbe peggio, p. I47).

Nel complesso, il volume contiene contributi estremamente interessanti, 
che non riescono però ad integrarsi in una costruzione organica; la storia degli studi scivola troppe volte in secondo piano rispetto a considerazioni di natura più "tradizionalmente" archeologica, che dovrebbero essere estranee al tema di questo volume. Il lettore ne deriva una quantità notevole di spunti e stimoli - lo studio sistematico sul ruolo di Rodi nella memoria culturale europea e sulla storia degli scavi sull'isola a partire da questo specifico contesto culturale, però, è ancora ampiamente da scrivere.

\section{Filippo CARLÀ-UHINK Universität Potsdam} filippo.carla-uhink@uni-potsdam.de

Vasiliki Zachari, Élise Lehoux et Noémie Hosor (dir.), La cité des regards. Autour de François Lissarrague, Rennes, Presses universitaires de Rennes, 20I9, 3 o6 p., 3о є/ ISBN 9782753576094 .

C'est un précieux ouvrage qu'ont dirigé Vasiliki Zachari, Élise Lehoux et Noémie Hosoi en hommage à leur professeur François Lissarrague, dont les travaux menés depuis plus de trente-cinq ans en font une figure tutélaire pour tout chercheur qui s'intéresse à l'iconographie et la culture grecques. C'est ainsi qu'après un court avant-propos, le livre s'ouvre sur une bibliographie complète du spécialiste de l'anthropologie des images et sur une introduction de V. Azoulay et de Fl. Gherchanoc qui retrace le "parcours en images» du directeur de l'EHESS justifiant le titre même qui paraphrase le catalogue collectif La cité des images: religion et société en Grèce antique, préfacé par J.-P. Vernant (Paris et Lausanne, Fernand Nathan et L.E.P., I984) dont un passage majeur est rappelé : “ Lire une des images implique aussi qu'on se soit fait le regard grec en s'efforçant de pénétrer le code visuel qui conférait aux multiples figures, pour les yeux d'un contemporain, leur immédiate lisibilité »(p. 28). Cette introduction revient sur l'historiographie de la lecture anthropologique de l'image dans la Grèce antique et permet de rappeler les axes de recherche de Fr. Lissarague et son approche de l'iconographique antique, à savoir les questions de la polysémie et de la spécificité du langage des images, de leur contextualisation et de leurs fonctions, de la mise en œuvre de jeux visuels et des rapports entre les images et leurs supports, autant de thématiques qui donnent à voir l'homme dans son rapport au monde, à la société, à la culture et à l'imaginaire qui s'y rattache.

Le rappel de ces problématiques donne son articulation à l'étude organisée en cinq parties en écho au propos introductif. La première «De l'objet à son dessin au $\mathrm{xIX}^{\mathrm{e}}$ siècle” comprend deux articles. Le premier, d'É. Lehoux, présente les Kunstmythologie conservées à l'Institut Winckelmann et leur rôle dans l'enseignement et les méthodes d'analyse des images de l'archéologie au xix ${ }^{\mathrm{e}}$ siècle, tandis que M.-A. Bernard analyse, dans le second, trois dessins de deux vases antiques de manière à interroger la pertinence et l'intérêt de cette documentation graphique pour l'étude matérielle des objets. La deuxième partie est consacrée aux « rituels au filtre de l'image » dans la lignée des travaux de Fr. Lissarrague sur les figures $\mathrm{du}$ guerrier et du satyre notamment. G. Deschodt, à travers l'exemple de scènes de mariage, établit l'importance de la mise en séries des images et de la nécessité de les regarder dans leurs relations au support et à l'ensemble du décor pour en comprendre les significations. B. Perriello étudie le personnage mythologique de Parthénopée, fils d'Atalante, afin d'envisager la polysémie de cette figure de l'entre-deux qu'est l'éphèbe dans la société grecque. F. Mazari compare l'iconographie de la purification des Prö̈tides et d'Oreste et en analyse le 
rituel social, ce qui l'amène à conclure sur un langage visuel paradigmatique, permettant une identification immédiate par le public de ce rétablissement de l'ordre social. L'article de M. Pedrina est dédié à un cratère paestan représentant la supplication d'Alcmène lue comme rituel de passage par sa mise en série à d'autres céramiques italiotes et aux sources textuelles. La troisième partie interroge la polysémie des figures mythologiques. Ainsi I. Sforza interroge-t-elle les attributs d'Apollon Hyperpontios sur l'hydrie du Vatican i6568 dans leur spécificité symbolique par rapport aux autres descriptions textuelles ou figurées, alors que Ph. Arnaud interprète une amphore de Vulci connue par un seul dessin comme lecture synchronique des âges de la vie du guerrier, dont les représentations de Diomède triomphant, Ajax blessé et Achille mort sont emblématiques. A. Callaud questionne, quant à elle, l'iconographie des déesses ailées dans la céramique attique à figures noires pour en souligner la place intermédiaire et la dimension presque héraldique qui signifie la présence et l'intervention du divin dans la sphère des humains. H. Collard prolonge cette problématique de la représentation des relations entre dieux et hommes par l'analyse des scènes de sacrifice sur l'autel peintes sur des pinakes de Locres. Celles-ci s'expliquent par la fonction votive de l'objet et montrent en même temps un décalage ludique par la présence du satyre, qui confère une dimension métaphorique et spirituelle à l'image en associant sacrifice et mariage. Après l'approche polysémique des images, celle de leur contexte est abordée dans la quatrième partie. N. Strawczynski étudie le motif de la poursuite amoureuse et plus précisément les objets qui chutent au cours de ces épisodes comme formes symboliques du “Temps d'Éros », temps éphémère et furtif du désir et de la beauté de la jeunesse. N. Kéi envisage, quant à elle, à partir d'une analogie avec le décor végétal, le poulpe comme motif ornemental polyvalent et polysémique, dont la forme polymorphe est la traduction visuelle de la mêtis, ses « mille plis » devenant le signe des “mille ruses». M. Baggio propose ensuite une lecture inédite d'un cratère du Museo Nazionale de Tarente représentant Polynice et Ériphyle, en observant le rôle des objets figurés, un coffret, un collier et un métier à tisser, comme vecteurs de sens en tant qu'instruments de séduction et de tromperie. Un dernier article d'E. Reshetnikova questionne les contradictions interprétatives du type de l'amant solitaire avec un lièvre vu tantôt comme éromène tantôt comme éraste, ce qui implique une étude minutieuse du contexte et l'hypothèse d'un statut ambivalent de cette figure intermédiaire. Enfin, la cinquième partie est dédiée aux images fragmentées. V. Zachari interroge l'espace vide comme potentiel degré zéro de l'image à travers l'exemple d'un corpus de vases à motif cultuel sans présence humaine, pour conclure que ce degré zéro n'existe pas et que le sens de l'image se construit par l'association de l'objet à d'autres éléments - végétaux, architecturaux ou ornementaux - pour dire implicitement le rituel par les empreintes humaines. N. Dietrich prolonge ces réflexions heuristiques en se consacrant à la notion de fragmentarité à partir de l'analyse d'un tesson d'une coupe de Douris figurant les plis d'un chiton et d'un himation et les pieds d'un personnage, afin de souligner la valeur heuristique de ce fragment dans la façon de regarder les objets, puisqu'il implique de prendre en considération les détails et de se focaliser sur un élément partiel, ce qui suscite de nouvelles interrogations à mettre en regard des objets complets et contextes mieux connus.

Enfin, une conclusion rappelle l'objectif du livre, pleinement atteint, de donner “l'envie de poursuivre les pistes initiées par F. Lissarrague » (p. 29I) grâce à ses contributions axées sur la notion de l'entre-deux de 
l'image, ses jeux et ses significations. C'est donc un ouvrage d'une grande richesse qui donne matière à penser, à regarder et à revoir aux historiens, historiens de l'art, archéologues, anthropologues et sémiologues de l'image, qu'ils soient spécialistes de l'Antiquité ou d'une autre période, tant par les problématiques abordées que par les cas d'étude traités qui peuvent ouvrir de nombreuses perspectives quant à l'anthropologie et la sémiologie de ces « images-objets » que sont les céramiques grecques (sur la notion d'، images-objets, cf. J. Baschet, L'iconographie médiévale, Paris, Gallimard, 2008, p. 33-34 et p. 6o-6I).

Clarisse Evrard

Université de Lille clarisse.evrard@univ-lille.fr

Sandra Zanella, La caccia fu buona : pour une histoire des fouilles à Pompéi de Titus à l'Europe, Naples, Centre Jean Bérard, 20I9, 366 p., $42 €$ / ISBN 97829I88878I2.

Consacré à l'histoire des fouilles de Pompéiàtravers les archives archéologiques, l'ouvrage de Sandra Zanella (SZ) trouve ses origines dans la première partie d'une thèse intitulée «Bâtiments privés sur sol public? Les maisons en terrasse de Pompéi entre le forum et les murailles ", soutenue en 20I5. Le titre, inspiré d'un propos tenu en I769 par Joseph II, rappelle au lecteur que l'exploration de Pompéi se résuma parfois à une chasse au bel objet. Si le sous-titre évoque une histoire de la redécouverte de la cité vésuvienne dans son ensemble, le propos tend en réalité rapidement à se concentrer sur l'îlot 2 de la regio VIII une plateforme surplombant la plaine du Sarno, entre la basilique du forum et le temple de Vénus. Ce secteur situé sur l'une des principales routes d'accès au plateau à l'époque moderne se révèle, au fil des pages, particulièrement propice à l'écriture d'une histoire de la seconde vie de Pompéi, comme l'auteur le démontre de manière convaincante. Cette seconde vie regroupe à la fois la réoccupation du plateau de Civita et le long processus de redécouverte de la cité antique par les fouilles. SZ rassemble notamment la documentation archéologique liée à la découverte en I936 dans la casa di Championnet I d'une structure témoignant de cette réoccupation post-éruptive : bien qu'il ne soit pas daté, ce four bâti à partir de matériaux antiques réemployés repose en effet au-dessus des niveaux liés à l'éruption d'octobre 79 ap.J.-C. Le choix de l'îlot VIII 2 permet également d'envisager l'histoire des fouilles: les pentes de ce secteur situé entre le centre monumental et une route au sud ont été alternativement recouvertes par les déblais de fouilles de zones plus prestigieuses (forum, casa del Fauno) et elles-mêmes objet de fouilles dans une succession de dégagements / ensevelissements méthodiquement reconstituée par SZ grâce à la documentation technique ancienne et à sa maîtrise de la bibliographie pompéienne la plus récente.

Le principal mérite du travail de SZ est en effet de proposer pour la première fois la micro-histoire d'un secteur de Pompéi sur la longue durée, ce qui lui permet de renouveler notre connaissance globale de la ville. Pour n'en donner qu'un exemple, plusieurs indices amènent SZ à supposer que la partie située au sud du forum avait déjà servi à l'évacuation des matériaux lors de l'opération de récupération de blocs et de statues supervisée par deux anciens consuls sous le règne de Titus. Ce faisant, elle s'inscrit dans la lignée des travaux pionniers de F. Zevi, de G. Soricelli, V. Scarano Ussani ou A. Storchi Marino sur les curatores restituendae Campaniae mais cela l'amène, par ricochet, à fragiliser la vision imposée par les travaux d'A. Maiuri selon laquelle l'état de délabrement du centre monumental de Pompéi aurait résulté du tremblement de terre de 63 . 
N'évitant pas toujours les redites, le plan de l'ouvrage souffre d'un déséquilibre qui ne nuit pas à l'intérêt de ses démonstrations et à l'importance de ses apports. L'auteur s'attache, dans la première partie (52 p.), à rassembler les éléments relatifs à la seconde vie de Pompéi de 79 jusqu'en I799 avant d'exploiter ensuite les archives de fouilles pour écrire l'histoire de la redécouverte du secteur VIII 2 et contribuer ainsi à une histoire plurielle de Pompéi (89 p.). La dernière partie (32 p.), consacrée à l'historiographie des travaux sur les quatre maisons de l'îlot en question, s'intègre sans doute moins bien au propos général de l'ouvrage qu'à l'économie de la thèse qui l'a inspiré. On apprécie particulièrement l'ampleur donnée aux annexes (I36 p.), véritables pièces justificatives qui retranscrivent très utilement des extraits de journaux de fouille manuscrits et de correspondance administrative (les références de la pièce 2 ont été omises). Précédé d'une liste des normes éditoriales de transcription adoptées, ce travail très précieux qui donne accès à la documentation primaire est illustré par la reproduction de plusieurs dessins et plans indiquant la dénomination des espaces employée par les fouilleurs, même si SZ les a par ailleurs convertis dans le système usuel de désignation des îlots mis en place par G. Fiorelli.

D’une manière générale, la richesse et la qualité de l'illustration sont très appréciables avec i 73 figures (en noir et blanc ou couleur) dont plusieurs dépliants à trois volets accompagnés de 3 planches dues à l'auteure. Le potentiel documentaire de ces illustrations est indéniable, qu'il s'agisse des plans anciens, de croquis de carnets de fouille, de relevés ou de photographies d'époque. Les documents les plus anciens sont particulièrement mis en lumière dans le propos comme dans la mise en page. En revanche, les clichés de fouille figurant entre les p. 257 et 271 sont très peu lisibles (certains ne dépassent pas
$4 \mathrm{~cm}$ de largeur), de même que le nuancier de la légende de la planche III qui gêne la lecture de la chronologie du dégagement de Pompéi. Les scories de relecture sont très rares : les figures 33 et 125 (manifeste pour l'adjudication des fouilles de I8II) sont en doublon, on lira "point » au lieu de " signe ferme » (p. 198) et “ hai trovato » au lieu de “ ahi trovato» (p. 283). La bibliographie, très complète, rendra d'utiles services aux chercheurs peu familiers de la cité pompéienne. Deux ouvrages, mentionnés en note de bas de page, manquent toutefois à la bibliographie générale (Cazanove 2008 ; Ling 1997).

Ces remarques de détail n'enlèvent rien à l'excellente tenue de l'ouvrage et à son intérêt scientifique qui tient notamment dans la volonté de SZ de fonder ses analyses sur l'étude la plus exhaustive et la plus approfondie possible de la documentation ancienne, plutôt que de se contenter d'une sélection de documents aisément consultables. Cette démarche prévient le risque de donner une vision biaisée des fouilles de Pompéi qui furent un modèle scruté par toute l'Europe antiquaire avant même la naissance d'une archéologie scientifique.

Vivien BARRIÈre Cergy Paris université vivien.barriere@cyu.fr

\section{Donna Zuckerberg, Not All Dead White Men. Classics andMisogyny in the Digital Age, Cambridge et Londres, Harvard University Press, 20I8, 288 p., \$27,95 / ISBN 9780674975552.}

En raison de sa quête identitaire, l'extrême droite a toujours instrumentalisé ses " racines » antiques. Dans Not All Dead White Men: Classics and Misogyny in the Digital Age, publié en 2018 par Harvard University Press, Donna Zuckerberg se 
penche sur un aspect contemporain de ce type de réception: la lecture et l'instrumentalisation de l'Antiquité, notamment des stoïciens et d'Ovide, sur les forums en ligne et dans les blogs masculinistes américains. Auteure d'une thèse sur Aristophane et Euripide soutenue à Stanford et directrice de la revue numérique Eidolon consacrée à la culture classique et ses rapports avec l'actualité, l'auteure connaît bien et le monde antique et le monde numérique contemporain. Elle explique avoir écrit son livre en réaction à la vague de misogynie sur internet et au harcèlement dont elle et d'autres féministes ont été l'objet par la “manosphère», néologisme qui désigne les communautés en ligne majoritairement composées d'hommes d'extrême droite misogynes. L'élection de Donald Trump peu après qu'elle a terminé la rédaction de son ouvrage donne à celui-ci un nouvel écho.

L'auteure adopte une démarche pédagogique pour s'adresser aussi bien aux féministes peu au fait de la culture classique qu'aux spécialistes de l'Antiquité pas nécessairement sensibilisés aux questions du féminisme. Tout en consacrant un utile développement aux supports des sphères numériques concernées, elle souligne la complexité et la diversité des discours qui y sont tenus, mais semble néanmoins faire un peu trop l'impasse sur les réseaux sociaux pour se consacrer aux blogs et aux forums; dans l'index, Twitter apparaît trois fois, pour des mentions anecdotiques, Facebook pas du tout (sur la famille de Donna Zuckerberg et ses relations avec son frère Mark, PDG de Facebook, lire Nosheen Iqbal, “ Donna Zuckerberg: 'Social media has elevated misogyny to new levels of violence' ", The Guardian, II/I/2018. En ligne : https://www.theguardian.com/books/20I8/ nov/II/donna-zuckerberg-social-mediamisoyny-violence-classical-antiquity-notall-dead-white-men). Les manifestations de l'alt-right états-unienne étant sensiblement différentes de celles qu'on trouve dans le contexte français voire européen, ces précisions préliminaires permettent au lectorat français de se familiariser avec le contexte américain. L'essai se termine par un glossaire définissant les termes utilisés par la manosphere comme alpha male (un homme socialement dominant et attrayant), incel (involontairement célibataire) ou hypergamy (tendance féminine à rechercher comme partenaire les hommes au statut social le plus élevé, aussi appelée gold-digging).

Le matériel analysé provient majoritairement de la communauté numérique “The Red Pill » et d'articles publiés par ses membres. Hébergé sur la plateforme Reddit, qui rencontre un succès moindre en Europe qu'aux États-Unis, cet espace doit son nom à la pilule rouge avalée par le héros du film Matrix (I999), qui lui permet d'échapper à l'ignorance bienheureuse dans laquelle il baignait pour prendre conscience de la réalité de son monde. Les 230.0oo membres de cette communauté en ligne (lors de la publication du volume), en majorité des hommes hétérosexuels blancs, considèrent que la société occidentale est en proie au féminisme et subit, en dépit des apparences, la domination féminine. Liés par leur haine des femmes et leur racisme, ils semblent issus de divers courants d'extrême droite. Que vient faire l'Antiquité classique dans ces discours misogynes? Comme le démontre Donna Zuckerberg, le renvoi à celle-ci fonde une argumentation d'autorité susceptible d'être instrumentalisée aussi bien d'un point de vue humaniste et progressiste qu'à des fins conservatrices. En l'occurrence, la culture classique fournit aux membres de “The Red Pill » des exempla, des outils de réflexion et d'argumentation pour défendre le patriarcat. L'étude décrypte comment les philosophes antiques, Épictète et Marc Aurèle en tête, sont instrumentalisés pour alimenter une tension entre hommes et femmes et justifier aussi bien l'infériorité de ces dernières que leur viol. 
Le premier chapitre de l'ouvrage présente le contexte politique et rhétorique au sein duquel les classiques sont exploités, les raisons de cet usage et les problèmes que cela soulève. Les procédés de la manosphère pour convaincre son public et ses détracteurs sont décryptés en mettant notamment en avant la notion de frame control qui vise à déstabiliser une personne afin de lui faire adopter son point de vue. Les deux chapitres suivants s'arrêtent sur deux des thèmes classiques favoris des auteurs et membres de The Red Pill, montrant comment ceux-ci sélectionnent les textes qui les arrangent. Le chapitre 2 est consacré au stoïcisme envisagé comme un outil de développement personnel, une doctrine et un mode de vie pour former l'identité masculine. Le chapitre 3 concerne principalement L'art d'aimer d'Ovide, conçu comme un guide de séduction illustrant la permanence intemporelle des relations entre genres et justifiant une lecture flexible du concept de consentement féminin. En replaçant des extraits de The Red Pill dans une perspective historique, Donna Zuckerberg se livre à une analyse critique méthodique des interprétations que leurs auteurs proposent des sources antiques en argumentant scientifiquement. Tout en admettant les composantes misogynes de certains textes antiques mis en avant, elle les nuance et les réinscrit dans leur contexte, démontre les contradictions et la partialité de la lecture qui en est faite et en explique les tenants et aboutissants. De même qu'elle a expliqué à ses lecteurs, ou plutôt ses lectrices, les outils rhétoriques mis en œuvre par l'extrême droite, elle les arme d'arguments solides, dont l'efficacité dans le débat public n'est cependant pas assurée... En effet, comment renvoyer efficacement à la méthode historique pour répondre à un opposant faisant un usage idéologique assumé de l'histoire?

Le dernier chapitre, modestement intitulé How to Save Western Civilization, porte sur le patriarcat idéal nourri de philosophie antique revisitée tel que le rêvent les sous- cultures masculinistes d'extrême droite. En prétendant restaurer des modèles sociaux et familiaux anciens, la femme est envisagée comme une éternelle mineure privée de libre arbitre, dépendant des décisions d'un tuteur masculin plus éclairé. Il est longuement question des fausses accusations de viol et de l'ancienneté de ce mal à travers la figure de Phèdre, dont l'auteure souligne qu'elle est source de déchirements des milieux féministes et de prises de positions contradictoires sur l'étude des classiques: des modèles à connaître ou des repoussoirs à condamner ?

Assumé comme un plaidoyer en faveur d'une approche féministe et progressiste de la culture classique, Not All Dead White Men est un ouvrage important sur la situation actuelle des classics aux États-Unis. La réflexion aurait gagné à se placer dans une perspective plus globale et internationale, en prenant notamment en compte les réseaux sociaux par le biais desquels les membres de The Red Pill recrutent et harcèlent. Donna Zuckerberg tire la sonnette d'alarme et invite les spécialistes de l'Antiquité à s'emparer de la problématique. Non seulement aux ÉtatsUnis, mais aussi en Europe où, selon des modalités un peu différentes, l'idéologie masculiniste est nourrie par les populismes et nationalismes ambiants.

Plan du volume: Introduction (p.I); I. Arms and the Manosphere (p. II); 2. The Angriest Stoics (p. 45); 3. The Ovid Method (p. 89); 4. How to Save Western Civilization (p. 143); Conclusion (p. I85); Glossary of Red Pill Terms (p. I9I); Notes (p. I95); References (p. 229); Acknowledgments (p. 253); Index (p. 255-27o).

Fabien Bit̀vre-Perrin Centre Jean Bérard (EfR, CNRS) fabienbp@hotmail.com 
